\title{
POLYNOMIAL CALCULATIONS IN DOPPLER TRACKING
}

\author{
TSUNG-LIN LEE* ${ }^{*}$ SONG-SUN LIN ${ }^{\dagger}$, WEN-WEI LIN${ }^{\ddagger}$, SHING-TUNG YAU ${ }^{\S}$, \\ AND JUBO ZHU
}

\begin{abstract}
Tracking a moving object by the Doppler effect is an important tool to locate the position and to measure the velocity of a moving object. In theory, the corresponding movement of the object can be formulated by a system of 12 quadratic polynomials in 12 unknowns. In this paper, we mainly propose a novel simplification to reduce the original system to a system of 4 polynomials of degrees 4, 3, 2 and 2 in 4 unknowns. Furthermore, we can also reduce the original system to a new system of only three quadratic polynomials in 3 unknowns when the 6 observation stations are located at the vertices and centre of a regular pentagon, numerical experiments show that the simplified polynomial system can be solved by the homotopy method efficiently and reliably. The method is much more robust than Newton's method when the initial vector is far from the solution. Also, the regular pentagon case outperforms the other configurations in terms of numerical accuracy.
\end{abstract}

1. Introduction. Tracking of moving objects is an important subject in many applications $[2,3,9]$. In general, the relative positions of moving objects are unknown and to be determined. Nevertheless, the relative speed of a moving object is known and can be measured by the Doppler effect by some observation stations. For tracking an object in the space, we are interested in finding its position $\mathbf{u}(t)=(x(t), y(t), z(t))^{T}$ and the associated velocity $\dot{\mathbf{u}}(t)=(\dot{x}(t), \dot{y}(t), \dot{z}(t))^{T}$ in time $t$. Suppose we have $\mathrm{N}$ observation stations located at $\left\{\mathbf{s}_{j} \equiv\left(x_{j}, y_{j}, z_{j}\right)^{T}\right\}_{j=1}^{N}$. The distances between $\mathbf{u}(t)$ and $\mathbf{s}_{j}$, and the associated derivatives, can be formulated, for $j=1, \ldots, N$, as

$$
\begin{gathered}
\left(x-x_{j}\right)^{2}+\left(y-y_{j}\right)^{2}+\left(z-z_{j}\right)^{2}=r_{j}^{2}, \\
\left(x-x_{j}\right) \dot{x}+\left(y-y_{j}\right) \dot{y}+\left(z-z_{j}\right) \dot{z}=r_{j} \dot{r}_{j} .
\end{gathered}
$$

In practice, the data $\dot{r}_{j}$ in (2) can be measured by the Doppler effect.

By substituting $r_{j}=\sqrt{\left(x-x_{j}\right)^{2}+\left(y-y_{j}\right)^{2}+\left(z-z_{j}\right)^{2}}$ of (1) into (2), we get

$$
F_{j}(\mathbf{v})=\frac{\left(x-x_{j}\right) \dot{x}+\left(y-y_{j}\right) \dot{y}+\left(z-z_{j}\right) \dot{z}}{\sqrt{\left(x-x_{j}\right)^{2}+\left(y-y_{j}\right)^{2}+\left(z-z_{j}\right)^{2}}}-\dot{r}_{j}=0, \quad(j=1, \ldots, N)
$$

*Department of Applied Mathematics, National Sun Yat-sen University, Kaohsiung 80424, Taiwan, ROC; E-mail: leetsung@math.nsysu.edu.tw

${ }^{\dagger}$ Department of Applied Mathematics, National Chiao Tung University, Hsinchu 30010, Taiwan, ROC; E-mail: sslin@math.nctu.edu.tw

${ }_{\ddagger}^{\ddagger}$ Department of Applied Mathematics, National Chiao Tung University, Hsinchu 30010, Taiwan, ROC; E-mail: wwlin@math.nctu.edu.tw

$\S$ Department of Mathematics, Harvard University, Cambridge, MA 02138, USA; E-mail: yau@math.harvard.edu

ฯ College of Science, National University of Defence Technology, Changsha 410073, China; E-mail: ju_bo_zhu@yahoo.com.cn 
where $\mathbf{v}=(x, y, z, \dot{x}, \dot{y}, \dot{z})^{T}$. Since $\mathbf{v}$ has 6 unknowns, it is natural to consider $N=$ 6 in (3) with 6 measured data $\left\{\dot{r}_{j}\right\}_{j=1}^{6}$ and a system of nonlinear equations $F=$ $\left(F_{1}, \cdots, F_{6}\right)^{T}: \mathbb{R}^{6} \longrightarrow \mathbb{R}^{6}$. Newton's method can then be applied for its solution.

In practice, there are advantages and disadvantages for solving (3) by Newtontype methods. It is well-known that Newton's method converges locally and quadratically. However, to locate a "good" initial vector, in general, is crucial. It may become difficult if the time step in the observation process is too large (see the numerical experiments in Section 4). In addition, equations in (3) are fully nonlinear, thus the evaluation of the Jacobian matrix in each Newton step may be costly. Therefore, in the following sections, we are motivated to reconsider solving the original system of polynomials in (1) and (2) in the 12 unknowns $\left\{x, y, z, \dot{x}, \dot{y}, \dot{z}, r_{1}, \cdots, r_{6}\right\}$.

Historical Note. The problem of using six stations to track a moving object was proposed by the last author Zhu and his group. Newton's method were applied, with its associated problems of initial guesses. In December 2011, Zhu asked Yau whether the problem can be solved in closed form. Yau proposed the elimination of variables, reducing the problem to the intersection of a couple polynomials. The detail and nontrivial analysis was then carried out by the first three authors, and the calculations were implemented by the fast polynomial system solver developed by TY Li (Michigan State University).

2. Simplification of Systems of Polynomials. The homotopy method $[1,6]$ is a very powerful tool for finding all solutions of systems of polynomial equations. We now consider the original system of quadratic polynomials of (1) and (2) with $N=6$ in 12 unknowns $\left\{x, y, z, \dot{x}, \dot{y}, \dot{z}, r_{1}, \cdots, r_{6}\right\}$, and propose a novel simplification to reduce the 12 unknowns to three or four unknowns so that the homotopy method can be applied more efficiently.

For given 6 positions $\left\{\left(x_{j}, y_{j}, z_{j}\right)^{T}\right\}_{j=1}^{6}$ of observation stations, and the measured speeds $\left\{\dot{r}_{j}\right\}_{j=1}^{6}$ by the Doppler effect in (1) and (2), we denote the following simplifying notations. Let

(4) $\quad V_{0}=\left[\begin{array}{ccc}x_{1} & y_{1} & z_{1} \\ x_{2} & y_{2} & z_{2} \\ x_{3} & y_{3} & z_{3} \\ x_{4} & y_{4} & z_{4} \\ x_{5} & y_{5} & z_{5} \\ x_{6} & y_{6} & z_{6}\end{array}\right], \mathbf{n}=\left[\begin{array}{c}x_{1}^{2}+y_{1}^{2}+z_{1}^{2} \\ x_{2}^{2}+y_{2}^{2}+z_{2}^{2} \\ x_{3}^{2}+y_{3}^{2}+z_{3}^{2} \\ x_{4}^{2}+y_{4}^{2}+z_{4}^{2} \\ x_{5}^{2}+y_{5}^{2}+z_{5}^{2} \\ x_{6}^{2}+y_{6}^{2}+z_{6}^{2}\end{array}\right], \quad \dot{\mathbf{r}}=\left[\begin{array}{c}\dot{r}_{1} \\ \dot{r}_{2} \\ \dot{r}_{3} \\ \dot{r}_{4} \\ \dot{r}_{5} \\ \dot{r}_{6}\end{array}\right], \quad \mathbf{r}=\left[\begin{array}{c}r_{1} \\ r_{2} \\ r_{3} \\ r_{4} \\ r_{5} \\ r_{6}\end{array}\right]$, 
(5)

$$
\dot{R}=\operatorname{diag}(\dot{\mathbf{r}}), \quad R=\operatorname{diag}(\mathbf{r}), \quad \mathbf{u}=\left[\begin{array}{c}
x \\
y \\
z
\end{array}\right], \quad \dot{\mathbf{u}}=\left[\begin{array}{c}
\dot{x} \\
\dot{y} \\
\dot{z}
\end{array}\right], \quad \mathbf{r} \circ \mathbf{r}=\left[\begin{array}{c}
r_{1}^{2} \\
r_{2}^{2} \\
r_{3}^{2} \\
r_{4}^{2} \\
r_{5}^{2} \\
r_{6}^{2}
\end{array}\right]
$$

Subtracting the 1 st equation from the $j$ th equation $(j \neq 1)$ in $(2)$ yields

$$
C V_{0} \dot{\mathbf{u}}=-C \dot{R} \mathbf{r}, \quad C=\left[\begin{array}{cccccc}
-1 & 1 & 0 & 0 & 0 & 0 \\
-1 & 0 & 1 & 0 & 0 & 0 \\
-1 & 0 & 0 & 1 & 0 & 0 \\
-1 & 0 & 0 & 0 & 1 & 0 \\
-1 & 0 & 0 & 0 & 0 & 1
\end{array}\right]
$$

Similar manipulations in (1) produce

$$
C V_{0} \mathbf{u}=\frac{1}{2} C(\mathbf{n}-\mathbf{r} \circ \mathbf{r})
$$

Assuming that $C V_{0}$ is of full rank, the matrix $V_{0}^{T} C^{T} C V_{0}$ is invertible. Multiplying (6) and (7) by $V_{0}^{T} C^{T}$, respectively, we have

$$
\dot{\mathbf{u}}=\left(V_{0}^{T} C^{T} C V_{0}\right)^{-1}\left(V_{0}^{T} C^{T} C\right)(-\dot{R} \mathbf{r})
$$

and

$$
\mathbf{u}=\frac{1}{2}\left(V_{0}^{T} C^{T} C V_{0}\right)^{-1}\left(V_{0}^{T} C^{T} C\right)(\mathbf{n}-\mathbf{r} \circ \mathbf{r})
$$

It is easily seen that the unknowns $\mathbf{u}=(x, y, z)^{T}$ and $\dot{\mathbf{u}}=(\dot{x}, \dot{y}, \dot{z})^{T}$ in (8) and (9) can be represented in terms of $\left\{r_{j}\right\}_{j=1}^{6}$. Denote, for $j=1, \ldots, 6$,

$$
\begin{gathered}
A=\left(V_{0}^{T} C^{T} C V_{0}\right)^{-1}\left(V_{0}^{T} C^{T} C\right), \quad \mathbf{u}_{j}=\left(x_{j}, y_{j}, z_{j}\right)^{T}, \\
\mathbf{p}=(-\dot{R} \mathbf{r}) \equiv\left(p_{1}, \ldots, p_{6}\right)^{T}, \quad \mathbf{q}=\frac{1}{2}(\mathbf{n}-\mathbf{r} \circ \mathbf{r}) .
\end{gathered}
$$

Substituting equations (8) and (9) into (2) and (1), we have, for $j=1, \ldots, 6$, the cubic equations

$$
\mathbf{q}^{T} A^{T} A \mathbf{p}-\mathbf{u}_{j}^{T} A \mathbf{p}=-p_{j},
$$

and the quartic equations

$$
\mathbf{q}^{T} A^{T} A \mathbf{q}-2 \mathbf{u}_{i}^{T} A \mathbf{q}+\mathbf{u}_{j}^{T} \mathbf{u}_{j}=\dot{r}_{j}^{-2} p_{j}^{2}
$$

in the 12 unknown $\left\{x, y, z, \dot{x}, \dot{y}, \dot{z}, r_{1}, \ldots, r_{6}\right\}$. 
We now subtract the 2 nd equation of (12) from the $j$ th equation for $j=3, \ldots, 6$ and get four equations

$$
\left(\mathbf{u}_{2}-\mathbf{u}_{j}\right)^{T} A \mathbf{p}=p_{2}-p_{j}
$$

Denote

$$
\widehat{C}=\left[\begin{array}{llllll}
0 & -1 & 1 & 0 & 0 & 0 \\
0 & -1 & 0 & 1 & 0 & 0 \\
0 & -1 & 0 & 0 & 1 & 0 \\
0 & -1 & 0 & 0 & 0 & 1
\end{array}\right]
$$

the equations in (14) can be rewritten in matrix form

$$
\left(\left[\begin{array}{c}
\left(\mathbf{u}_{2}-\mathbf{u}_{3}\right)^{T} \\
\left(\mathbf{u}_{2}-\mathbf{u}_{4}\right)^{T} \\
\left(\mathbf{u}_{2}-\mathbf{u}_{5}\right)^{T} \\
\left(\mathbf{u}_{2}-\mathbf{u}_{6}\right)^{T}
\end{array}\right] A+\widehat{C}\right) \dot{R} \mathbf{r} \equiv \widehat{A} \mathbf{r}=\mathbf{0}
$$

where $\widehat{A} \in \mathbb{R}^{4 \times 6}$. In fact, from the original system in (1), the rank of $\widehat{A}$ can be shown generically to be 2 (see Appendix A). Hence, let $Q^{T} \widehat{A}=\left[\begin{array}{cc}T_{1} & T_{2} \\ O & O\end{array}\right]$ be the QR factorization [4] of $\widehat{A}$, where $Q \in \mathbb{R}^{4 \times 4}$ is orthogonal, $T_{2} \in \mathbb{R}^{2 \times 4}$ and $T_{1} \in \mathbb{R}^{2 \times 2}$ is nonsingular. Then from (4) and (16), the unknowns $\left\{r_{1}, r_{2}\right\}$ can be represented in terms of $r_{3}, r_{4}, r_{5}$ and $r_{6}$ :

$$
\left[\begin{array}{l}
r_{1} \\
r_{2}
\end{array}\right]=-T_{1}^{-1} T_{2}\left[\begin{array}{l}
r_{3} \\
r_{4} \\
r_{5} \\
r_{6}
\end{array}\right]
$$

Therefore, from (8), (9) and (17), we can solve the cubic equations of (12) and the quartic equations of $(13)$ on the $\left(r_{3}, r_{4}, r_{5}, r_{6}\right)$-plane.

Next, we simplify equations of (12) and (13) with $j=1$. From (11) and the relation in (17), the 1st equation in (12) can be simplified to a cubic equations in $r_{3}, r_{4}, r_{5}$ and $r_{6}$ :

$$
(\mathbf{r} \circ \mathbf{r})^{T} \frac{1}{2} A^{T} A \dot{R} \mathbf{r}-\hat{\mathbf{u}}_{1}^{T} A \dot{R} \mathbf{r}=r_{1} \dot{r}_{1}, \quad \hat{\mathbf{u}}_{1}=\frac{1}{2} A \mathbf{n}-\mathbf{u}_{1} .
$$

Similarly, from (11), the 1st equation of (13) can be written as

$$
\begin{aligned}
& \mathbf{q}^{T} A^{T} A \mathbf{q}-2 \mathbf{u}_{1}^{T} A \mathbf{q}+\mathbf{u}_{1}^{T} \mathbf{u}_{1} \\
= & \frac{1}{4}(\mathbf{r} \circ \mathbf{r})^{T} A^{T} A(\mathbf{r} \circ \mathbf{r})-\left(\frac{1}{2} \mathbf{n}^{T} A^{T}-\mathbf{u}_{1}^{T}\right) A(\mathbf{r} \circ \mathbf{r}) \\
& +\left(\frac{1}{4} \mathbf{n}^{T} A^{T} A \mathbf{n}-\mathbf{u}_{1}^{T} A \mathbf{n}+\mathbf{u}_{1}^{T} \mathbf{u}_{1}\right) \\
= & r_{1}^{2} .
\end{aligned}
$$


From the definition $\hat{\mathbf{u}}_{1}$ in (18) and the relation in (17), (19) can be simplified to a quartic equation in $r_{3}, r_{4}, r_{5}$ and $r_{6}$ :

$$
\frac{1}{4}(\mathbf{r} \circ \mathbf{r})^{T} A^{T} A(\mathbf{r} \circ \mathbf{r})-\hat{\mathbf{u}}_{1}^{T} A(\mathbf{r} \circ \mathbf{r})+\hat{c}=r_{1}^{2},
$$

where $\hat{c}=\left(\frac{1}{4} \mathbf{n}^{T} A^{T} A \mathbf{n}-\mathbf{u}_{1}^{T} A \mathbf{n}+\mathbf{u}_{1}^{T} \mathbf{u}_{1}\right)$.

Finally, we subtract the 2 nd equation of (13) from the $j$ th equation for $j=3, \ldots, 6$ and get four equations

$$
2\left(\mathbf{u}_{2}-\mathbf{u}_{j}\right)^{T} A \mathbf{q}+\mathbf{u}_{j}^{T} \mathbf{u}_{j}-\mathbf{u}_{2}^{T} \mathbf{u}_{2}=r_{j}^{2}-r_{2}^{2} .
$$

From (16), (21) can be written in the matrix form

$$
\begin{gathered}
\left.\left(\left[\begin{array}{c}
\left(\mathbf{u}_{2}-\mathbf{u}_{3}\right)^{T} \\
\left(\mathbf{u}_{2}-\mathbf{u}_{4}\right)^{T} \\
\left(\mathbf{u}_{2}-\mathbf{u}_{5}\right)^{T} \\
\left(\mathbf{u}_{2}-\mathbf{u}_{6}\right)^{T}
\end{array}\right] A+\widehat{C}\right) \mathbf{r} \circ \mathbf{r}=\left(\begin{array}{c}
\left(\mathbf{u}_{2}-\mathbf{u}_{3}\right)^{T} \\
\left(\mathbf{u}_{2}-\mathbf{u}_{4}\right)^{T} \\
\left(\mathbf{u}_{2}-\mathbf{u}_{5}\right)^{T} \\
\left(\mathbf{u}_{2}-\mathbf{u}_{6}\right)^{T}
\end{array}\right] A+\widehat{C}\right) \mathbf{n} \\
\equiv \widehat{A} \mathbf{r} \circ \mathbf{r}=\widehat{A} \mathbf{n}
\end{gathered}
$$

The matrix $\widehat{A}$ is generically of rank 2 (see Appendix A). Hence, it is sufficient to choose two linearly independent equations in (22).

For given positions of stations $\left\{\left(x_{j}, y_{j}, z_{j}\right)^{T}\right\}_{j=1}^{6}$ and 6 measured data $\left\{\dot{r}_{j}\right\}_{j=1}^{6}$ by the Doppler effect, the radii $\left\{r_{j}\right\}_{j=1}^{6}$ can be computed by solving the system of 4 polynomials of degree $3,4,2$ and 2 in 4 unknowns $r_{3}, r_{4}, r_{5}$ and $r_{6}$ :

$$
\left\{\begin{array}{l}
\frac{1}{2}(\mathbf{r} \circ \mathbf{r})^{T} A^{T} A \dot{R} \mathbf{r}-\hat{\mathbf{u}}_{1}^{T} A \dot{R} \mathbf{r}-\dot{r}_{1} r_{1}=0 \\
\frac{1}{4}(\mathbf{r} \circ \mathbf{r})^{T} A^{T} A(\mathbf{r} \circ \mathbf{r})-\hat{\mathbf{u}}_{1}^{T} A(\mathbf{r} \circ \mathbf{r})+\hat{c}-r_{1}^{2}=0 \\
\left(\mathbf{u}_{2}-\mathbf{u}_{3}\right)^{T} A(\mathbf{r} \circ \mathbf{r})-r_{2}^{2}+r_{3}^{2}-\mathbf{u}_{3}^{T} \mathbf{u}_{3}+\mathbf{u}_{2}^{T} \mathbf{u}_{2}-\left(\mathbf{u}_{2}-\mathbf{u}_{3}\right)^{T} A \mathbf{n}=0 \\
\left(\mathbf{u}_{2}-\mathbf{u}_{4}\right)^{T} A(\mathbf{r} \circ \mathbf{r})-r_{2}^{2}+r_{4}^{2}-\mathbf{u}_{4}^{T} \mathbf{u}_{4}+\mathbf{u}_{2}^{T} \mathbf{u}_{2}-\left(\mathbf{u}_{2}-\mathbf{u}_{4}\right)^{T} A \mathbf{n}=0
\end{array}\right.
$$

where $\left[\begin{array}{ll}r_{1} & r_{2}\end{array}\right]^{T}=-T_{1}^{-1} T_{2}\left[\begin{array}{llll}r_{3} & r_{4} & r_{5} & r_{6}\end{array}\right]^{T}$. The system in (23) has 48 solutions. The position $(x, y, z)^{T}$ and the velocity $(\dot{x}, \dot{y}, \dot{z})^{T}$, respectively, of the moving object are immediately obtained by

$$
\left\{\begin{array}{l}
\mathbf{u}=(x, y, z)^{T}=\frac{1}{2} A(\mathbf{n}-\mathbf{r} \circ \mathbf{r}) \\
\dot{\mathbf{u}}=(\dot{x}, \dot{y}, \dot{z})^{T}=-A \dot{R} \mathbf{r}
\end{array}\right.
$$

Furthermore, (23) can theoretically be simplified to a system of 2 polynomial equations of degrees 12 and 16 in 2 unknowns. Compared to (23), this simplification reduces the number of unknowns from 4 to 2 . However, the number of potential solutions is increased from 48 to 192. In practice, the 2-variable polynomial system with higher degrees in much more troublesome than (23) when solved by the homotopy method. We leave the tedious derivation of this simplification in Appendix B. 
3. Special Configurations of Stations. In this section we will consider several particular configurations: stations on level ground or on a sphere. It is obvious that if the configuration of stations is collinear or degenerate (with some stations at identical coordinates), the polynomial system for the moving objects is over-determined. Throughout this paper, we may assume that the stations are not collinear or degenerate.

3.1. Stations on level ground. With all stations on level ground, the original system in (1) and (2) becomes, for $j=1, \ldots, 6$,

$$
\begin{gathered}
\left(x-x_{j}\right)^{2}+\left(y-y_{j}\right)^{2}+(z-0)^{2}=r_{j}^{2}, \\
\left(x-x_{j}\right) \dot{x}+\left(y-y_{j}\right) \dot{y}+(z-0) \dot{z}=r_{j} \dot{r}_{j},
\end{gathered}
$$

where $\left\{\left(x_{j}, y_{j}\right)^{T}, \dot{r}_{j}\right\}_{j=1}^{6}$ are the given data and $\left\{x, y, z, \dot{x}, \dot{y}, \dot{z}, r_{1}, \cdots, r_{6}\right\}$ are the unknowns.

With this particular configuration we will show that the system of (25) and (26) can be simplified to a system of 3 quadratic polynomials in 3 unknowns. Furthermore, if all stations are on a circle, the polynomial equations will be degenerated. Later we also consider some special configurations, with 6 stations forming: (i) a regular pentagon and its centre, and (ii) two regular triangles. For convenience, we denote, for $j=1, \cdots, 6$,

$$
V_{0}=\left[\begin{array}{ll}
x_{1} & y_{1} \\
x_{2} & y_{2} \\
x_{3} & y_{3} \\
x_{4} & y_{4} \\
x_{5} & y_{5} \\
x_{6} & y_{6}
\end{array}\right], \quad \mathbf{n}=\left[\begin{array}{c}
x_{1}^{2}+y_{1}^{2} \\
x_{2}^{2}+y_{2}^{2} \\
x_{3}^{2}+y_{3}^{2} \\
x_{4}^{2}+y_{4}^{2} \\
x_{5}^{2}+y_{5}^{2} \\
x_{6}^{2}+y_{6}^{2}
\end{array}\right], \quad \mathbf{u}=\left[\begin{array}{c}
x \\
y
\end{array}\right], \quad \dot{\mathbf{u}}=\left[\begin{array}{c}
\dot{x} \\
\dot{y}
\end{array}\right], \quad \mathbf{u}_{j}=\left[\begin{array}{c}
x_{j} \\
y_{j}
\end{array}\right] .
$$

The notations $\mathbf{r}, \mathbf{r} \circ \mathbf{r}, R, \dot{\mathbf{r}}, \dot{R}$ and $C$ are the same as in Section 2 .

As the derivation in (8) and (9), we also have

$$
\begin{aligned}
\dot{\mathbf{u}} & =-\left(V_{0}^{T} C^{T} C V_{0}\right)^{-1}\left(V_{0}^{T} C^{T} C\right)(\dot{R} \mathbf{r}) \\
& \equiv-A \dot{R} \mathbf{r}
\end{aligned}
$$

and

$$
\mathbf{u}=\frac{1}{2} A(\mathbf{n}-\mathbf{r} \circ \mathbf{r})
$$

where $V_{0}$ and $\mathbf{n}$ are given in (27). From (28) and (29) we see that the unknown$\mathrm{s}\{x, y, \dot{x}, \dot{y}\}$ can be represented in terms of $\left\{r_{j}\right\}_{j=1}^{6}$. Recall that $\mathbf{p}=(-\dot{R} \mathbf{r}) \equiv$ 
$\left(p_{1}, \ldots, p_{6}\right)^{T}$ and $\mathbf{q}=(\mathbf{n}-\mathbf{r} \circ \mathbf{r}) / 2$ as in (11). Substituting (28) and (29) into (25) and (26), we get, for $j=1, \ldots, 6$, the cubic equations

$$
\mathbf{q}^{T} A^{T} A \mathbf{p}-\mathbf{u}_{j}^{T} A \mathbf{p}+z \dot{z}=-p_{j}
$$

and the quartic equations

$$
\mathbf{q}^{T} A^{T} A \mathbf{q}-2 \mathbf{u}_{j}^{T} A \mathbf{q}+\mathbf{u}_{j}^{T} \mathbf{u}_{j}+z^{2}=\dot{r}_{j}^{-2} p_{j}^{2} .
$$

We now subtract the 1st equation of (30) from the rest, yielding for $j=2, \ldots, 6$ :

$$
\left(\mathbf{u}_{1}-\mathbf{u}_{j}\right)^{T} A \mathbf{p}=p_{1}-p_{j} .
$$

The equations in (32) can be rewritten in matrix form

$$
\left(\left[\begin{array}{c}
\left(\mathbf{u}_{1}-\mathbf{u}_{2}\right)^{T} \\
\left(\mathbf{u}_{1}-\mathbf{u}_{3}\right)^{T} \\
\left(\mathbf{u}_{1}-\mathbf{u}_{4}\right)^{T} \\
\left(\mathbf{u}_{1}-\mathbf{u}_{5}\right)^{T} \\
\left(\mathbf{u}_{1}-\mathbf{u}_{6}\right)^{T}
\end{array}\right] A+C\right) \dot{R} \mathbf{r} \equiv \tilde{A} \mathbf{r}=\mathbf{0},
$$

where $\tilde{A} \in \mathbb{R}^{5 \times 6}$. When the original system is of the form as in (25), $\tilde{A}$ is generically of rank 3 . Hence, it is sufficient to choose three linearly independent equations in (33), say the first three equations, to from the system

$$
\left(\left[\begin{array}{c}
\left(\mathbf{u}_{1}-\mathbf{u}_{2}\right)^{T} \\
\left(\mathbf{u}_{1}-\mathbf{u}_{3}\right)^{T} \\
\left(\mathbf{u}_{1}-\mathbf{u}_{4}\right)^{T}
\end{array}\right] A+\widetilde{C}\right) \dot{R} \mathbf{r} \equiv \widetilde{B} \mathbf{r}=\mathbf{0}
$$

where $\widetilde{C}=\left[\begin{array}{cccccc}-1 & 1 & 0 & 0 & 0 & 0 \\ -1 & 0 & 1 & 0 & 0 & 0 \\ -1 & 0 & 0 & 1 & 0 & 0\end{array}\right]$ and $\widetilde{B} \in \mathbb{R}^{3 \times 6}$ is of full row rank. Let $Q^{T} \widetilde{B}=$ $\left[\begin{array}{ll}T_{1} & T_{2}\end{array}\right]$ be the QR decomposition of $\widetilde{B}$, where $Q \in \mathbb{R}^{3 \times 3}$ is orthogonal, $T_{2} \in \mathbb{R}^{3 \times 3}$ and $T_{1} \in \mathbb{R}^{3 \times 3}$ is nonsingular. Thus, $r_{1}, r_{2}, r_{3}$ can be represented in terms of $r_{4}, r_{5}$ and $r_{6}$ as

$$
\left[\begin{array}{l}
r_{1} \\
r_{2} \\
r_{3}
\end{array}\right]=-T_{1}^{-1} T_{2}\left[\begin{array}{l}
r_{4} \\
r_{5} \\
r_{6}
\end{array}\right]
$$

Therefore, we can solve the cubic equations of (30) and quartic equations of (31) on the $\left(r_{4}, r_{5}, r_{6}\right)$-plane.

Next, we subtract the 1 st equation of (31) from the $j$ th equation for $j=2, \ldots, 6$ and get five equations

$$
2\left(\mathbf{u}_{1}-\mathbf{u}_{j}\right)^{T} A \mathbf{q}+\mathbf{u}_{j}^{T} \mathbf{u}_{j}-\mathbf{u}_{1}^{T} \mathbf{u}_{1}=r_{j}^{2}-r_{1}^{2} .
$$


The equations in (36) can be written in the matrix form

$$
\widetilde{A} \mathbf{r} \circ \mathbf{r}=\widetilde{A} \mathbf{n}
$$

where $\widetilde{A}$ is defined by (33). As in (34) we also have $\widetilde{B}(\mathbf{r} \circ \mathbf{r})=\widetilde{B} \mathbf{n}$.

Given the data $\left\{\left(x_{j}, y_{j}\right)^{T}, \dot{r}_{j}\right\}_{j=1}^{6}$, the radii $\left\{r_{j}\right\}_{j=1}^{6}$ can be computed by solving the system of 3 quadratic polynomials in 3 unknowns $r_{4}, r_{5}$ and $r_{6}$ :

$$
\left\{\begin{array}{l}
\left(\mathbf{u}_{6}-\mathbf{u}_{1}\right)^{T} A(\mathbf{r} \circ \mathbf{r})-r_{6}^{2}+r_{1}^{2}-\mathbf{u}_{1}^{T} \mathbf{u}_{1}+\mathbf{u}_{6}^{T} \mathbf{u}_{6}-\left(\mathbf{u}_{6}-\mathbf{u}_{1}\right)^{T} A \mathbf{n}=0 \\
\left(\mathbf{u}_{6}-\mathbf{u}_{2}\right)^{T} A(\mathbf{r} \circ \mathbf{r})-r_{6}^{2}+r_{2}^{2}-\mathbf{u}_{2}^{T} \mathbf{u}_{2}+\mathbf{u}_{6}^{T} \mathbf{u}_{6}-\left(\mathbf{u}_{6}-\mathbf{u}_{2}\right)^{T} A \mathbf{n}=0 \\
\left(\mathbf{u}_{6}-\mathbf{u}_{3}\right)^{T} A(\mathbf{r} \circ \mathbf{r})-r_{6}^{2}+r_{3}^{2}-\mathbf{u}_{3}^{T} \mathbf{u}_{3}+\mathbf{u}_{6}^{T} \mathbf{u}_{6}-\left(\mathbf{u}_{6}-\mathbf{u}_{3}\right)^{T} A \mathbf{n}=0
\end{array}\right.
$$

where $\left[\begin{array}{lll}r_{1} & r_{2} & r_{3}\end{array}\right]^{T}=-T_{1}^{-1} T_{2}\left[\begin{array}{lll}r_{4} & r_{5} & r_{6}\end{array}\right]^{T}$ as in (35). The system (38) has potentially 8 solutions. The position $\mathbf{u}=(x, y, z)^{T}$ and the velocity $\dot{\mathbf{u}}=(\dot{x}, \dot{y}, \dot{z})^{T}$ of the moving object are immediately obtained, respectively, by (25) and (29), as well as, (26) and (28).

On the other hand, the system (38) can also be simplified to one equation of degree 8 with one unknown. Expanding the first 2 equations in (38) yields

$$
\left\{\begin{array}{l}
a_{4} r_{4}^{2}+a_{5} r_{5}^{2}+a_{6} r_{6}^{2}+a_{45} r_{4} r_{5}+a_{46} r_{4} r_{6}+a_{56} r_{5} r_{6}+a_{0}=0, \\
b_{4} r_{4}^{2}+b_{5} r_{5}^{2}+b_{6} r_{6}^{2}+b_{45} r_{4} r_{5}+b_{46} r_{4} r_{6}+b_{56} r_{5} r_{6}+b_{0}=0 .
\end{array}\right.
$$

We can rewrite them in the quadratic form: $\widetilde{\mathbf{r}}^{T} A_{0} \widetilde{\mathbf{r}}+a_{0}=0$ and $\widetilde{\mathbf{r}}^{T} B_{0} \widetilde{\mathbf{r}}+b_{0}=0$, where

$$
A_{0}=\left[\begin{array}{ccc}
a_{4} & \frac{a_{45}}{2} & \frac{a_{46}}{2} \\
\frac{a_{45}}{2} & a_{5} & \frac{a_{56}}{2} \\
\frac{a_{46}}{2} & \frac{a_{56}}{2} & a_{6}
\end{array}\right], \quad B_{0}=\left[\begin{array}{ccc}
b_{4} & \frac{b_{45}}{2} & \frac{b_{46}}{2} \\
\frac{b_{45}}{2} & b_{5} & \frac{b_{56}}{2} \\
\frac{b_{46}}{2} & \frac{b_{56}}{2} & b_{6}
\end{array}\right]
$$

and $\widetilde{\mathbf{r}}=\left[\begin{array}{lll}r_{4} & r_{5} & r_{6}\end{array}\right]^{T}$. Since $A_{0}$ and $B_{0}$ are symmetric, we assume that there is a nonsingular $V_{0}$ such that

$$
V_{0}^{T} A_{0} V_{0}=\left[\begin{array}{ccc}
\widetilde{a}_{4} & 0 & 0 \\
0 & \widetilde{a}_{5} & 0 \\
0 & 0 & \widetilde{a}_{6}
\end{array}\right], \quad V_{0}^{T} B_{0} V_{0}=\left[\begin{array}{ccc}
\widetilde{b}_{4} & 0 & 0 \\
0 & \widetilde{b}_{5} & 0 \\
0 & 0 & \widetilde{b}_{6}
\end{array}\right] .
$$

By changing variables

$$
\left[\begin{array}{l}
r_{4} \\
r_{5} \\
r_{6}
\end{array}\right]=V_{0}\left[\begin{array}{l}
s_{4} \\
s_{5} \\
s_{6}
\end{array}\right]
$$

the system (39) becomes

$$
\left\{\begin{array}{c}
\widetilde{a}_{4} s_{4}^{2}+\widetilde{a}_{5} s_{5}^{2}+\widetilde{a}_{6} s_{6}^{2}+a_{0}=0 \\
\widetilde{b}_{4} s_{4}^{2}+\widetilde{b}_{5} s_{5}^{2}+\widetilde{b}_{6} s_{6}^{2}+b_{0}=0
\end{array}\right.
$$


Assume that $\left[\begin{array}{cc}\widetilde{a}_{4} & \widetilde{a}_{5} \\ \widetilde{b}_{4} & \widetilde{b}_{5}\end{array}\right]$ in (42) is invertible. Then we have the following simple relations

$$
\left\{\begin{array}{l}
s_{4}^{2}=c_{1} s_{6}^{2}+c_{2} \\
s_{5}^{2}=c_{3} s_{6}^{2}+c_{4}
\end{array}\right.
$$

where $\left[\begin{array}{cc}c_{1} & c_{2} \\ c_{3} & c_{4}\end{array}\right]=\left[\begin{array}{cc}\widetilde{a}_{4} & \widetilde{a}_{5} \\ \widetilde{b}_{4} & \widetilde{b}_{5}\end{array}\right]^{-1}\left[\begin{array}{cc}\widetilde{a}_{6} & a_{0} \\ \widetilde{b}_{6} & b_{0}\end{array}\right]$. Recall that the last equation in (38) is a quadratic polynomial in variables $r_{4}, r_{5}$ and $r_{6}$. Plugging (41) into (38), the degrees of new equations remain the same. In fact, each of them can be written as

$$
s_{4} g_{1}+s_{5} g_{2}=s_{4} s_{5} g_{3}+g_{4},
$$

where $g_{1}, g_{2}, g_{3}$ and $g_{4}$ are polynomials in $s_{4}, s_{5}$ and $s_{6}$, and the exponents of $s_{4}$ and $s_{5}$ in $g_{i}$ 's are even numbers. Squaring both sides yields

$$
s_{4}^{2} g_{1}^{2}+s_{5}^{2} g_{2}^{2}+2 g_{1} g_{2} s_{4} s_{5}=s_{4}^{2} s_{5}^{2} g_{3}^{2}+2 g_{3} g_{4} s_{4} s_{5}+g_{4}^{2} .
$$

Collecting $s_{4} s_{5}$ on one side, we have $2\left(g_{1} g_{2}-g_{3} g_{4}\right) s_{4} s_{5}=s_{4}^{2} s_{5}^{2} g_{3}^{2}+g_{4}^{2}-s_{4}^{2} g_{1}^{2}-s_{5}^{2} g_{2}^{2}$. Squaring both sides of the new equation results in an equation which has variables $s_{4}, s_{5}$ and $s_{6}$, and the exponents of $s_{4}$ and $s_{5}$ are even numbers in all terms. Using (43), the equation only involves the variable $s_{6}$. Therefore, the system (38) can be simplified to one equation of degree 8 with even power terms being nonzero.

Remark (i) For this case, we can reduce(30), (31) to one equation in one variable $s_{6}$ of degree 8 which can be solved by QR algorithm [4] efficiently. However, it has too many possibilities to transform the solutions $s_{6}$ back to $r_{4}, r_{5}$ and $r_{6}$ by (39) because of the repeated squaring of (44). This is the drawback of this simplification. The other one is the eigendecomposition in (38) may not always exist. In practice, we do not recommend the simplification of (40).

(ii) When all stations are on a circle, the vector $\mathbf{n}$ is parallel to $(1, \cdots, 1)^{T}$. In this case, the right hand side of (38) is a zero vector, and (41) is a system of 3 homogeneous equations of degree 2 which is degenerated.

Example (Regular Pentagon): Given five stations lying at

$$
\left\{\left(h \cos \frac{2 \pi k}{5}, h \sin \frac{2 \pi k}{5}, 0\right)^{T} \mid k=0,1,2,3,4\right\}
$$

and the other one at origin $(0,0,0)^{T}$. The equation (36) gives the relations

$$
\left\{\begin{array}{l}
r_{1}=\frac{5+\sqrt{5}}{10} \frac{\dot{r}_{4}}{\dot{r}_{1}} r_{4}-\frac{\sqrt{5}}{5} \frac{\dot{r}_{5}}{\dot{r}_{1}} r_{5}+\frac{5+\sqrt{5}}{10} \frac{\dot{r}_{6}}{\dot{r}_{1}} r_{6}, \\
r_{2}=\frac{\dot{r}_{4}}{\dot{r}_{2}} r_{4}-\frac{1+\sqrt{5}}{2} \frac{\dot{r}_{5}}{\dot{r}_{2}} r_{5}+\frac{1+\sqrt{5}}{2} \frac{\dot{r}_{6}}{\dot{r}_{2}} r_{6}, \\
r_{3}=\frac{1+\sqrt{5}}{2} \frac{\dot{r}_{4}}{\dot{r}_{3}} r_{4}-\frac{1+\sqrt{5}}{2} \frac{\dot{r}_{5}}{\dot{r}_{3}} r_{5}+\frac{\dot{r}_{6}}{\dot{r}_{3}} r_{6},
\end{array}\right.
$$


and the equation of (38) are equivalent to

$$
\left\{\begin{aligned}
f_{1} \equiv & \frac{1}{10}\left(10 h^{2}+10 r_{1}^{2}-6 r_{2}^{2}-r_{3}^{2}+\sqrt{5} r_{3}^{2}-r_{4}^{2}-\sqrt{5} r_{4}^{2}\right. \\
& \left.-r_{5}^{2}-\sqrt{5} r_{5}^{2}-r_{6}^{2}+\sqrt{5} r_{6}^{2}\right)=0, \\
f_{2} \equiv & \frac{1}{10}\left(10 h^{2}+10 r_{1}^{2}-r_{2}^{2}+\sqrt{5} r_{2}^{2}-6 r_{3}^{2}-r_{4}^{2}+\sqrt{5} r_{4}^{2}\right. \\
& \left.-r_{5}^{2}-\sqrt{5} r_{5}^{2}-r_{6}^{2}-\sqrt{5} r_{6}^{2}\right)=0, \\
f_{3} \equiv & \frac{1}{10}\left(10 h^{2}+10 r_{1}^{2}-r_{2}^{2}-\sqrt{5} r_{2}^{2}-r_{3}^{2}+\sqrt{5} r_{3}^{2}-6 r_{4}^{2}\right. \\
& \left.-r_{5}^{2}+\sqrt{5} r_{5}^{2}-r_{6}^{2}-\sqrt{5} r_{6}^{2}\right)=0 .
\end{aligned}\right.
$$

Plugging (46) into (47), we have a system of 3 quadratic polynomials in variables $r_{4}, r_{5}$, and $r_{6}$ :

$$
\left[q_{1}, q_{2}, q_{3}\right]^{T}=0
$$

where

$$
\begin{aligned}
q_{1} \equiv & \frac{1}{10}\left(10 h^{2}-r_{4}^{2}-\sqrt{5} r_{4}^{2}-r_{5}^{2}-\sqrt{5} r_{5}^{2}-r_{6}^{2}+\sqrt{5} r_{6}^{2}\right. \\
& -\frac{\left((1+\sqrt{5}) r_{4} \dot{r}_{4}-(1+\sqrt{5}) r_{5} \dot{r}_{5}+2 r_{6} \dot{r}_{6}\right)^{2}}{4 \dot{r}_{3}^{2}} \\
& +\frac{\sqrt{5}\left((1+\sqrt{5}) r_{4} \dot{r}_{4}-(1+\sqrt{5}) r_{5} \dot{r}_{5}+2 r_{6} \dot{r}_{6}\right)^{2}}{4 \dot{r}_{3}^{2}} \\
& +\frac{\left((5+\sqrt{5}) r_{4} \dot{r}_{4}-2 \sqrt{5} r_{5} \dot{r}_{5}+(5+\sqrt{5}) r_{6} \dot{r}_{6}\right)^{2}}{10 \dot{r}_{1}^{2}} \\
& \left.-\frac{3\left(-2 r_{4} \dot{r}_{4}+(1+\sqrt{5})\left(r_{5} \dot{r}_{5}-r_{6} \dot{r}_{6}\right)\right)^{2}}{2 \dot{r}_{2}^{2}}\right), \\
q_{2} \equiv & \frac{1}{10}\left(10 h^{2}-r_{4}^{2}+\sqrt{5} r_{4}^{2}-r_{5}^{2}-\sqrt{5} r_{5}^{2}-r_{6}^{2}-\sqrt{5} r_{6}^{2}\right. \\
& -\frac{3\left((1+\sqrt{5}) r_{4} \dot{r}_{4}-(1+\sqrt{5}) r_{5} \dot{r}_{5}+2 r_{6} \dot{r}_{6}\right)^{2}}{2 \dot{r}_{3}^{2}} \\
& +\frac{\left((5+\sqrt{5}) r_{4} \dot{r}_{4}-2 \sqrt{5} r_{5} \dot{r}_{5}+(5+\sqrt{5}) r_{6} \dot{r}_{6}\right)^{2}}{10 \dot{r}_{1}^{2}} \\
& -\frac{\left(-2 r_{4} \dot{r}_{4}+(1+\sqrt{5})\left(\mathrm{r} 5 \dot{r}_{5}-r_{6} \dot{r}_{6}\right)\right)^{2}}{4 \dot{r}_{2}^{2}} \\
& \left.+\frac{\sqrt{5}\left(-2 r_{4} \dot{r}_{4}+(1+\sqrt{5})\left(r_{5} \dot{r}_{5}-r_{6} \dot{r}_{6}\right)\right)^{2}}{4 \dot{r}_{2}^{2}}\right),
\end{aligned}
$$




$$
\begin{aligned}
q_{3} \equiv & \frac{1}{10}\left(10 h^{2}-6 r_{4}^{2}-r_{5}^{2}+\sqrt{5} r_{5}^{2}-r_{6}^{2}-\sqrt{5} r_{6}^{2}\right. \\
& -\frac{\left((1+\sqrt{5}) r_{4} \dot{r}_{4}-(1+\sqrt{5}) r_{5} \dot{r}_{5}+2 r_{6} \dot{r}_{6}\right)^{2}}{4 \dot{r}_{3}^{2}} \\
& +\frac{\sqrt{5}\left((1+\sqrt{5}) r_{4} \dot{r}_{4}-(1+\sqrt{5}) r_{5} \dot{r}_{5}+2 r_{6} \dot{r}_{6}\right)^{2}}{4 \dot{r}_{3}^{2}} \\
& +\frac{\left((5+\sqrt{5}) r_{4} \dot{r}_{4}-2 \sqrt{5} r_{5} \dot{r}_{5}+(5+\sqrt{5}) r_{6} \dot{r}_{6}\right)^{2}}{10 \dot{r}_{1}^{2}} \\
& -\frac{\left(-2 r_{4} \dot{r}_{4}+(1+\sqrt{5})\left(r_{5} \dot{r}_{5}-r_{6} \dot{r}_{6}\right)\right)^{2}}{4 \dot{r}_{2}^{2}} \\
& \left.-\frac{\sqrt{5}\left(-2 r_{4} \dot{r}_{4}+(1+\sqrt{5})\left(r_{5} \dot{r}_{5}-r_{6} \dot{r}_{6}\right)\right)^{2}}{4 \dot{r}_{2}^{2}}\right) .
\end{aligned}
$$

Given the Doppler data $\left\{\dot{r}_{j} \mid j=1, \ldots, 6\right\}$, the system of (48) has 8 solutions in general and can be solved by the total degree homotopy method very fast $(<0.01 \mathrm{sec}$.).

Example (Two Regular Triangles): Given three stations lying at

$$
\left\{\left(h \cos \frac{2 \pi k}{3}, h \sin \frac{2 \pi k}{3}, 0\right) \mid k=0,1,2\right\}
$$

and the other three lying at $\left\{\left(2 h \cos \frac{2 \pi k}{3}, 2 h \sin \frac{2 \pi k}{3}, 0\right) \mid k=0,1,2\right\}$. The equation (35) gives the relations

$$
\left\{\begin{array}{l}
r_{1}=-2 \frac{\dot{r}_{4}}{\dot{r}_{1}} r_{4}+\frac{\dot{r}_{5}}{\dot{r}_{1}} r_{5}+2 \frac{\dot{r}_{6}}{\dot{r}_{1}} r_{6} \\
r_{2}=5 \frac{\dot{r}_{4}}{\dot{r}_{2}} r_{4}-3 \frac{\dot{r}_{5}}{\dot{r}_{2}} r_{5}-\frac{\dot{r}_{6}}{\dot{r}_{2}} r_{6} \\
r_{3}=8 \dot{\underline{r}}_{3} r_{4}-5 \frac{\dot{r}_{5}}{\dot{r}_{3}} r_{5}-2 \frac{\dot{r}_{6}}{\dot{r}_{3}} r_{6}
\end{array}\right.
$$

and the equations of (38) are equivalent to

$$
\left\{\begin{array}{l}
f_{1} \equiv \frac{1}{315}\left(-1035 h^{2}+60 r_{1}^{2}-107 r_{2}^{2}+111 r_{3}^{2}-233 r_{4}^{2}+174 r_{5}^{2}-5 r_{6}^{2}\right)=0 \\
f_{2} \equiv \frac{1}{5}\left(-2 r_{2}^{2}+r_{3}^{2}+2 r_{4}^{2}-r_{5}^{2}\right)=0 \\
f_{3} \equiv \frac{1}{315}\left(-1305 h^{2}+240 r_{1}^{2}-113 r_{2}^{2}+129 r_{3}^{2}+13 r_{4}^{2}+66 r_{5}^{2}-335 r_{6}^{2}\right)=0
\end{array}\right.
$$

Plugging (49) into (50), we have a system of 3 quadratic polynomials in variables 
$r_{4}, r_{5}$, and $r_{6}$ :

$$
\begin{aligned}
q_{1} \equiv & -1035 h^{2}-233 r_{4}^{2}+174 r_{5}^{2}-5 r_{6}^{2}-\frac{107\left(-5 r_{4} \dot{r}_{4}+3 r_{5} \dot{r}_{5}+r_{6} \dot{r}_{6}\right)^{2}}{\dot{r}_{2}^{2}} \\
& +\frac{60\left(-2 r_{4} \dot{r}_{4}+r_{5} \dot{r}_{5}+2 r_{6} \dot{r}_{6}\right)^{2}}{\dot{r}_{1}^{2}}+\frac{111\left(-8 r_{4} \dot{r}_{4}+5 r_{5} \dot{r}_{5}+2 r_{6} \dot{r}_{6}\right)^{2}}{\dot{r}_{3}^{2}}=0, \\
(51) q_{2} \equiv & 2 r_{4}^{2}-r_{5}^{2}-\frac{2\left(-5 r_{4} \dot{r}_{4}+3 r_{5} \dot{r}_{5}+r_{6} \dot{r}_{6}\right)^{2}}{\dot{r}_{2}^{2}}+\frac{\left(-8 r_{4} \dot{r}_{4}+5 r_{5} \dot{r}_{5}+2 r_{6} \dot{r}_{6}\right)^{2}}{\dot{r}_{3}^{2}}=0, \\
q_{3} \equiv & -1305 h^{2}+13 r_{4}^{2}+66 r_{5}^{2}-335 r_{6}^{2}-\frac{113\left(-5 r_{4} \dot{r}_{4}+3 r_{5} \dot{r}_{5}+r_{6} \dot{r}_{6}\right)^{2}}{\dot{r}_{2}^{2}} \\
& +\frac{240\left(-2 r_{4} \dot{r}_{4}+r_{5} \dot{r}_{5}+2 r_{6} \dot{r}_{6}\right)^{2}}{\dot{r}_{1}^{2}}+\frac{129\left(-8 r_{4} \dot{r}_{4}+5 r_{5} \dot{r}_{5}+2 r_{6} \dot{r}_{6}\right)^{2}}{\dot{r}_{3}^{2}}=0 .
\end{aligned}
$$

Given the Doppler data $\left\{\dot{r}_{j} \mid j=1, \ldots, 6\right\}$, the system of (51) has 8 solutions and can also be solved by the total degree homotopy method very efficient.

3.2. Stations on a Sphere. When stations lie on a sphere, the vector $\mathbf{n}$ is parallel to $\left[\begin{array}{llllll}1 & 1 & 1 & 1 & 1 & 1\end{array}\right]^{T}$. Hence, the original system can be simplified to the system

$$
\left\{\begin{array}{l}
\frac{1}{2}(\mathbf{r} \circ \mathbf{r})^{T} A^{T} A \dot{R} \mathbf{r}+\mathbf{u}_{1}^{T} A \dot{R} \mathbf{r}-\dot{r}_{1} r_{1}=0 \\
\frac{1}{4}(\mathbf{r} \circ \mathbf{r})^{T} A^{T} A(\mathbf{r} \circ \mathbf{r})+\mathbf{u}_{1}^{T} A(\mathbf{r} \circ \mathbf{r})+\mathbf{u}_{1}^{T} \mathbf{u}_{1}-r_{1}^{2}=0 \\
\left(\mathbf{u}_{2}-\mathbf{u}_{3}\right)^{T} A(\mathbf{r} \circ \mathbf{r})-r_{2}^{2}+r_{3}^{2}=0 \\
\left(\mathbf{u}_{2}-\mathbf{u}_{4}\right)^{T} A(\mathbf{r} \circ \mathbf{r})-r_{2}^{2}+r_{4}^{2}=0
\end{array}\right.
$$

where $\left[\begin{array}{ll}r_{1} & r_{2}\end{array}\right]^{T}=-T_{1}^{-1} T_{2}\left[\begin{array}{llll}r_{3} & r_{4} & r_{5} & r_{6}\end{array}\right]^{T}$ as in (17). Given the Doppler data $\left\{\dot{r}_{j} \mid j=1, \ldots, 6\right\}$, the system of $(52)$ has 16 solutions in general.

Example (Regular Octahedron): Given six stations lying at

$$
\{( \pm h, 0,0),(0, \pm h, 0),(0,0, \pm h)\}
$$

The equation (17) gives the relations

$$
\left\{\begin{array}{l}
r_{1}=\frac{\dot{r}_{3}}{\dot{r}_{1}} r_{3}-\frac{\dot{r}_{4}}{\dot{r}_{1}} r_{4}+\frac{\dot{r}_{6}}{\dot{r}_{1}} r_{6} \\
r_{2}=\frac{\dot{r}_{3}}{\dot{r}_{2}} r_{3}-\frac{\dot{r}_{5}}{\dot{r}_{2}} r_{5}+\frac{\dot{r}_{6}}{\dot{r}_{2}} r_{6},
\end{array}\right.
$$


and the equations of (52) are equivalent to

(54)

$$
\left\{\begin{aligned}
f_{1} \equiv & \frac{1}{128 h^{2}}\left(36 r_{1}^{3} \dot{r}_{1}-6 r_{1}\left(r_{2}^{2}+r_{3}^{2}+2 r_{4}^{2}+r_{5}^{2}+r_{6}^{2}\right) \dot{r}_{1}+17 r_{2}^{3} \dot{r}_{2}+r_{2} r_{3}^{2} \dot{r}_{2}+2 r_{2} r_{4}^{2} \dot{r}_{2}\right. \\
& -15 r_{2} r_{5}^{2} \dot{r}_{2}+r_{2} r_{6}^{2} \dot{r}_{2}+r_{2}^{2} r_{3} \dot{r}_{3}+17 r_{3}^{3} \dot{r}_{3}+2 r_{3} r_{4}^{2} \dot{r}_{3}+r_{3} r_{5}^{2} \dot{r}_{3}-15 r_{3} r_{6}^{2} \dot{r}_{3} \\
& +2 r_{2}^{2} r_{4} \dot{r}_{4}+2 r_{3}^{2} r_{4} \dot{r}_{4}+4 r_{4}^{3} \dot{r}_{4}+2 r_{4} r_{5}^{2} \dot{r}_{4}+2 r_{4} r_{6}^{2} \dot{r}_{4}-15 r_{2}^{2} r_{5} \dot{r}_{5}+r_{3}^{2} r_{5} \dot{r}_{5} \\
& +2 r_{4}^{2} r_{5} \dot{r}_{5}+17 r_{5}^{3} \dot{r}_{5}+r_{5} r_{6}^{2} \dot{r}_{5}+r_{2}^{2} r_{6} \dot{r}_{6}-15 r_{3}^{2} r_{6} \dot{r}_{6}+2 r_{4}^{2} r_{6} \dot{r}_{6}+r_{5}^{2} r_{6} \dot{r}_{6} \\
& +17 r_{6}^{3} \dot{r}_{6}-6 r_{1}^{2}\left(r_{2} \dot{r}_{2}+r_{3} \dot{r}_{3}+2 r_{4} \dot{r}_{4}+r_{5} \dot{r}_{5}+r_{6} \dot{r}_{6}\right) \\
& \left.-16 h^{2}\left(2 r_{1} \dot{r}_{1}+r_{2} \dot{r}_{2}+r_{3} \dot{r}_{3}+2 r_{4} \dot{r}_{4}+r_{5} \dot{r}_{5}+r_{6} \dot{r}_{6}\right)\right)=0 \\
f_{2} \equiv & \frac{1}{256 h^{2}}\left(256 h^{4}+36 r_{1}^{4}+17 r_{2}^{4}+2 r_{2}^{2} r_{3}^{2}+17 r_{3}^{4}+4 r_{2}^{2} r_{4}^{2}+4 r_{3}^{2} r_{4}^{2}+4 r_{4}^{4}-30 r_{2}^{2} r_{5}^{2}\right. \\
& +2 r_{3}^{2} r_{5}^{2}+4 r_{4}^{2} r_{5}^{2}+17 r_{5}^{4}+2 r_{2}^{2} r_{6}^{2}-30 r_{3}^{2} r_{6}^{2}+4 r_{4}^{2} r_{6}^{2}+2 r_{5}^{2} r_{6}^{2}+17 r_{6}^{4} \\
& -12 r_{1}^{2}\left(r_{2}^{2}+r_{3}^{2}+2 r_{4}^{2}+r_{5}^{2}+r_{6}^{2}\right) \\
& \left.-32 h^{2}\left(2 r_{1}^{2}+r_{2}^{2}+r_{3}^{2}+2 r_{4}^{2}+r_{5}^{2}+r_{6}^{2}\right)\right)=0 \\
f_{3} \equiv & \frac{r_{2}^{2}}{2}-\frac{r_{3}^{2}}{2}+\frac{r_{5}^{2}}{2}-\frac{r_{6}^{2}}{2}=0 \\
f_{4} \equiv & -\frac{3 r_{1}^{2}}{4}+\frac{5 r_{2}^{2}}{8}+\frac{r_{3}^{2}}{8}-\frac{3 r_{4}^{2}}{4}+\frac{5 r_{5}^{2}}{8}+\frac{r_{6}^{2}}{8}=0 .
\end{aligned}\right.
$$

Substituting (53) into (54), we have a system of 4 polynomials of degrees $3,4,2$ and 2 in unknowns $r_{3}, r_{4}, r_{5}$, and $r_{6}$ :

$$
\begin{aligned}
q_{1} \equiv & 3 r_{3}^{3}\left(2 \dot{r}_{2}^{2} \dot{r}_{3}^{3}+\dot{r}_{1}^{2} \dot{r}_{3}\left(\dot{r}_{2}^{2}+\dot{r}_{3}^{2}\right)\right)+4 r_{4}^{3} \dot{r}_{1}^{2} \dot{r}_{2}^{2} \dot{r}_{4}+2 r_{4} r_{5}^{2} \dot{r}_{1}^{2} \dot{r}_{2}^{2} \dot{r}_{4}+2 r_{4} r_{6}^{2} \dot{r}_{1}^{2} \dot{r}_{2}^{2} \dot{r}_{4}-12 r_{4}^{3} \dot{r}_{2}^{2} \dot{r}_{4}^{3} \\
& +8 r_{5}^{3} \dot{r}_{1}^{2} \dot{r}_{2}^{2} \dot{r}_{5}+2 r_{4} r_{5}^{2} \dot{r}_{1}^{2} \dot{r}_{4} \dot{r}_{5}^{2}-8 r_{5}^{3} \dot{r}_{1}^{2} \dot{r}_{5}^{3}-2 r_{4}^{2} r_{6} \dot{r}_{1}^{2} \dot{r}_{2}^{2} \dot{r}_{6}-5 r_{5}^{2} r_{6} \dot{r}_{1}^{2} \dot{r}_{2}^{2} \dot{r}_{6}+3 r_{6}^{3} \dot{r}_{1}^{2} \dot{r}_{2}^{2} \dot{r}_{6} \\
& +30 r_{4}^{2} r_{6} \dot{r}_{2}^{2} \dot{r}_{4}^{2} \dot{r}_{6}-4 r_{4} r_{5} r_{6} \dot{r}_{1}^{2} \dot{r}_{4} \dot{r}_{5} \dot{r}_{6}+19 r_{5}^{2} r_{6} \dot{r}_{1}^{2} \dot{r}_{5}^{2} \dot{r}_{6}+2 r_{4} r_{6}^{2} \dot{r}_{1}^{2} \dot{r}_{4} \dot{r}_{6}^{2}-24 r_{4} r_{6}^{2} \dot{r}_{2}^{2} \dot{r}_{4} \dot{r}_{6}^{2} \\
& -14 r_{5} r_{6}^{2} \dot{r}_{1}^{2} \dot{r}_{5} \dot{r}_{6}^{2}+3 r_{6}^{3} \dot{r}_{1}^{2} \dot{r}_{6}^{3}+6 r_{6}^{3} \dot{r}_{2}^{2} \dot{r}_{6}^{3}-16 h^{2} \dot{r}_{1}^{2} \dot{r}_{2}^{2}\left(r_{3} \dot{r}_{3}+r_{6} \dot{r}_{6}\right) \\
& +r_{3}^{2}\left(2 r_{4}\left(-12 \dot{r}_{2}^{2} \dot{r}_{3}^{2}+\dot{r}_{1}^{2}\left(\dot{r}_{2}^{2}+\dot{r}_{3}^{2}\right)\right) \dot{r}_{4}\right. \\
& \left.-14 r_{5} \dot{r}_{1}^{2} \dot{r}_{3}^{2} \dot{r}_{5}+r_{6}\left(18 \dot{r}_{2}^{2} \dot{r}_{3}^{2}+\dot{r}_{1}^{2}\left(-5 \dot{r}_{2}^{2}+9 \dot{r}_{3}^{2}\right)\right) \dot{r}_{6}\right) \\
& -2 r_{4}^{2} \dot{r}_{2}^{2} r_{3} \dot{r}_{3}\left(\dot{r}_{1}^{2}-15 \dot{r}_{4}^{2}\right)+r_{5}^{2} \dot{r}_{1}^{2} r_{3} \dot{r}_{3}\left(-5 \dot{r}_{2}^{2}+19 \dot{r}_{5}^{2}\right)-28 r_{5} r_{6} \dot{r}_{1}^{2} \dot{r}_{5} \dot{r}_{6} r_{3} \dot{r}_{3} \\
& -4 r_{4} \dot{r}_{4} r_{3} \dot{r}_{3}\left(r_{5} \dot{r}_{1}^{2} \dot{r}_{5}-r_{6}\left(\dot{r}_{1}^{2}-12 \dot{r}_{2}^{2}\right) \dot{r}_{6}\right)+r_{6}^{2} r_{3} \dot{r}_{3}\left(18 \dot{r}_{2}^{2} \dot{r}_{6}^{2}+\dot{r}_{1}^{2}\left(-5 \dot{r}_{2}^{2}+9 \dot{r}_{6}^{2}\right)\right)=0,
\end{aligned}
$$

$$
\begin{aligned}
q_{2} \equiv & 256 h^{4}+17 r_{3}^{4}+4 r_{3}^{2} r_{4}^{2}+4 r_{4}^{4}+2 r_{3}^{2} r_{5}^{2}+4 r_{4}^{2} r_{5}^{2}+17 r_{5}^{4} \\
& -30 r_{3}^{2} r_{6}^{2}+4 r_{4}^{2} r_{6}^{2}+2 r_{5}^{2} r_{6}^{2}+17 r_{6}^{4} \\
& +\frac{36\left(r_{3} \dot{r}_{3}-r_{4} \dot{r}_{4}+r_{6} \dot{r}_{6}\right)^{4}}{\dot{r}_{1}^{4}}+\frac{2 r_{3}^{2}\left(r_{3} \dot{r}_{3}-r_{5} \dot{r}_{5}+r_{6} \dot{r}_{6}\right)^{2}}{\dot{r}_{2}^{2}}+\frac{4 r_{4}^{2}\left(r_{3} \dot{r}_{3}-r_{5} \dot{r}_{5}+r_{6} \dot{r}_{6}\right)^{2}}{\dot{r}_{2}^{2}} \\
& -\frac{30 r_{5}^{2}\left(r_{3} \dot{r}_{3}-r_{5} \dot{r}_{5}+r_{6} \dot{r}_{6}\right)^{2}}{\dot{r}_{2}^{2}}+\frac{2 r_{6}^{2}\left(r_{3} \dot{r}_{3}-r_{5} \dot{r}_{5}+r_{6} \dot{r}_{6}\right)^{2}}{\dot{r}_{2}^{2}}+\frac{17\left(r_{3} \dot{r}_{3}-r_{5} \dot{r}_{5}+r_{6} \dot{r}_{6}\right)^{4}}{\dot{r}_{2}^{4}}
\end{aligned}
$$




$$
\begin{gathered}
-\frac{12\left(r_{3} \dot{r}_{3}-r_{4} \dot{r}_{4}+r_{6} \dot{r}_{6}\right)^{2}\left(r_{3}^{2}+2 r_{4}^{2}+r_{5}^{2}+r_{6}^{2}+\frac{\left(r_{3} \dot{r}_{3}-r_{5} \dot{r}_{5}+r_{6} \dot{r}_{6}\right)^{2}}{\dot{r}_{2}^{2}}\right)}{\dot{r}_{1}^{2}} \\
-32 h^{2}\left(r_{3}^{2}+2 r_{4}^{2}+r_{5}^{2}+r_{6}^{2}+\frac{2\left(r_{3} \dot{r}_{3}-r_{4} \dot{r}_{4}+r_{6} \dot{r}_{6}\right)^{2}}{\dot{r}_{1}^{2}}\right. \\
\left.+\frac{\left(r_{3} \dot{r}_{3}-r_{5} \dot{r}_{5}+r_{6} \dot{r}_{6}\right)^{2}}{\dot{r}_{2}^{2}}\right)=0 \\
q_{3} \equiv-r_{3}^{2}+r_{5}^{2}-r_{6}^{2}+\frac{\left(r_{3} \dot{r}_{3}-r_{5} \dot{r}_{5}+r_{6} \dot{r}_{6}\right)^{2}}{\dot{r}_{2}^{2}}=0 \\
q_{4} \equiv r_{3}^{2}-6 r_{4}^{2}+5 r_{5}^{2}+r_{6}^{2}-\frac{6\left(r_{3} \dot{r}_{3}-r_{4} \dot{r}_{4}+r_{6} \dot{r}_{6}\right)^{2}}{\dot{r}_{1}^{2}}+\frac{5\left(r_{3} \dot{r}_{3}-r_{5} \dot{r}_{5}+r_{6} \dot{r}_{6}\right)^{2}}{\dot{r}_{2}^{2}}=0 .
\end{gathered}
$$

\section{Numerical Experiments.}

4.1. Numerical Accuracy of Homotopy Method. In this section we will show the numerical accuracy of solving the original systems and the corresponding simplified systems by the homotopy continuation method [7], implemented in HOM4PS-2.0 (available at

http://www.math.nsysu.edu.tw/ leetsung/works/HOM4PS_soft.htm).

(Regular Pentagon): Given five stations located at

$$
\left\{\left(2 \cos \frac{2 \pi k}{5}, 2 \sin \frac{2 \pi k}{5}, 0\right)^{T} \mid k=0,1,2,3,4\right\}
$$

and the other one at origin $(0,0,0)^{T}$. We assume the object is at the position $(2,5,3)^{T}$ with velocity $(1,2,4)^{T}$. Then the measured Doppler data should be

$$
\begin{array}{ll}
\dot{r}_{1}=3.893314107138301 & \dot{r}_{4}=3.839796099826884 \\
\dot{r}_{2}=3.772968873135194 & \dot{r}_{5}=3.6039686843251832 \\
\dot{r}_{3}=4.32328941267566 & \dot{r}_{6}=3.552946442309747 .
\end{array}
$$

Hence, from (1) and (2) the original polynomial system is

$$
\left\{\begin{array}{l}
(x-0) \dot{x}+(y-0 \quad) \dot{y}+(z-0) \dot{z}=3.893314107138301 \cdot r_{1}, \\
\left(x-2 \cos \frac{0 \pi}{5}\right) \dot{x}+\left(y-2 \sin \frac{0 \pi}{5}\right) \dot{y}+(z-0) \dot{z}=3.772968873135194 \cdot r_{2}, \\
\left(x-2 \cos \frac{2 \pi}{5}\right) \dot{x}+\left(y-2 \sin \frac{2 \pi}{5}\right) \dot{y}+(z-0) \dot{z}=4.32328941267566 \cdot r_{3}, \\
\left(x-2 \cos \frac{4 \pi}{5}\right) \dot{x}+\left(y-2 \sin \frac{4 \pi}{5}\right) \dot{y}+(z-0) \dot{z}=3.839796099826884 \cdot r_{4}, \\
\left(x-2 \cos \frac{6 \pi}{5}\right) \dot{x}+\left(y-2 \sin \frac{6 \pi}{5}\right) \dot{y}+(z-0) \dot{z}=3.6039686843251832 \cdot r_{5}, \\
\left(x-2 \cos \frac{8 \pi}{5}\right) \dot{x}+\left(y-2 \sin \frac{8 \pi}{5}\right) \dot{y}+(z-0) \dot{z}=3.552946442309747 \cdot r_{6}, \\
(x-0 \quad)^{2}+(y-0 \quad)^{2}+(z-0)^{2}=r_{1}^{2} \\
\left(x-2 \cos \frac{0 \pi}{5}\right)^{2}+\left(y-2 \sin \frac{0 \pi}{5}\right)^{2}+(z-0)^{2}=r_{2}^{2} \\
\left(x-2 \cos \frac{2 \pi}{5}\right)^{2}+\left(y-2 \sin \frac{2 \pi}{5}\right)^{2}+(z-0)^{2}=r_{3}^{2} \\
\left(x-2 \cos \frac{4 \pi}{5}\right)^{2}+\left(y-2 \sin \frac{4 \pi}{5}\right)^{2}+(z-0)^{2}=r_{4}^{2} \\
\left(x-2 \cos \frac{6 \pi}{5}\right)^{2}+\left(y-2 \sin \frac{6 \pi}{5}\right)^{2}+(z-0)^{2}=r_{5}^{2} \\
\left(x-2 \cos \frac{8 \pi}{5}\right)^{2}+\left(y-2 \sin \frac{8 \pi}{5}\right)^{2}+(z-0)^{2}=r_{6}^{2}
\end{array}\right.
$$


and from (48) the simplified system is

$$
\left\{\begin{array}{l}
4-0.18046568496886645 r_{4}^{2}+0.817451608915785 r_{4} r_{5} \\
-1.3605999657065038 r_{5}^{2}-0.6263091782107895 r_{4} r_{6} \\
+2.0051504885645226 r_{5} r_{6}-0.7498101264961088 r_{6}^{2}=0 \\
4-0.4781808395962457 r_{4}^{2}+1.3463107868155073 r_{4} r_{5} \\
-0.9485546803221847 r_{5}^{2}-0.09134819115874843 r_{4} r_{6} \\
+0.2012702178430671 r_{5} r_{6}-0.0058130967013134555 r_{6}^{2}=0 \\
4-0.17058796355164318 r_{4}^{2}-0.052044655877640844 r_{4} r_{5} \\
-0.2531518755495662 r_{5}^{2}+0.23087742111668474 r_{4} r_{6} \\
+0.7033756928598706 r_{5} r_{6}-0.5553510918753413 r_{6}^{2}=0 .
\end{array}\right.
$$

Solving (55) by the polyhedral homotopy continuation method [5, 6], HOM4PS$2.0[7]$ takes 3.5 seconds and obtains solutions

$$
\begin{array}{ll}
x=1.9999999999999849 & r_{1}=6.1644140029689547 \\
y=4.9999999999999849 & r_{2}=5.8309518948452830 \\
z=2.9999999999999911 & r_{3}=4.5284361228902368 \\
\dot{x}=0.9999999999999950 & r_{4}=6.0594084619828887 \\
\dot{y}=2.0000000000000027 & r_{5}=7.7606598302495318 \\
\dot{z}=3.9999999999999987 & r_{6}=7.6517314622837613,
\end{array}
$$

which has 13 correct digits.

Solving (56) by the polyhedral homotopy continuation method, HOM4PS-2.0 takes less than 0.01 seconds and obtains the solution

$$
r_{4}=6.0594084619829323, \quad r_{5}=7.7606598302495700, \quad r_{6}=7.6517314622837826,
$$

which has 14 correct digits.

Note that HOM4PS-2.0 is implemented in a double precision floating point arithmetic system (i.e. 16 significant digits), and the exact solution of (55) is

$$
\begin{array}{llll}
x=2 & \dot{x}=1 & r_{1}=6.164414002968976 & r_{4}=6.059408461982912 \\
y=5 & \dot{y}=2 & r_{2}=5.830951894845301 & r_{5}=7.760659830249555 \\
z=3 & \dot{z}=4 & r_{3}=4.528436122890257 & r_{6}=7.651731462283781 .
\end{array}
$$

Next, we consider the moving object is 10 times farther with the same station configuration:

We assume the object is at the position $(20,50,30)^{T}$ with the velocity $(1,2,4)^{T}$. Then the measured Doppler data should be 


$$
\begin{array}{ll}
\dot{r}_{1}=3.893314107138301 & \dot{r}_{4}=3.906605581821401 \\
\dot{r}_{2}=3.9000674757995495 & \dot{r}_{5}=3.8641247950598454 \\
\dot{r}_{3}=3.9322710660446627 & \dot{r}_{6}=3.8598600586840734 .
\end{array}
$$

The corresponding original polynomial system is

$$
\left\{\begin{array}{l}
(x-0) \dot{x}+(y-0 \quad) \dot{y}+(z-0) \dot{z}=3.893314107138301 \cdot r_{1} \\
\left(x-2 \cos \frac{0 \pi}{5}\right) \dot{x}+\left(y-2 \sin \frac{0 \pi}{5}\right) \dot{y}+(z-0) \dot{z}=3.9000674757995495 \cdot r_{2} \\
\left(x-2 \cos \frac{2 \pi}{5}\right) \dot{x}+\left(y-2 \sin \frac{2 \pi}{5}\right) \dot{y}+(z-0) \dot{z}=3.9322710660446627 \cdot r_{3} \\
\left(x-2 \cos \frac{4 \pi}{5}\right) \dot{x}+\left(y-2 \sin \frac{4 \pi}{5}\right) \dot{y}+(z-0) \dot{z}=3.906605581821401 \cdot r_{4} \\
\left(x-2 \cos \frac{6 \pi}{5}\right) \dot{x}+\left(y-2 \sin \frac{6 \pi}{5}\right) \dot{y}+(z-0) \dot{z}=3.8641247950598454 \cdot r_{5} \\
\left(x-2 \cos \frac{8 \pi}{5}\right) \dot{x}+\left(y-2 \sin \frac{8 \pi}{5}\right) \dot{y}+(z-0) \dot{z}=3.8598600586840734 \cdot r_{6} \\
(x-0)^{2}+(y-0 \quad)^{2}+(z-0)^{2}=r_{1}^{2} \\
\left(x-2 \cos \frac{0 \pi}{5}\right)^{2}+\left(y-2 \sin \frac{0 \pi}{5}\right)^{2}+(z-0)^{2}=r_{2}^{2} \\
\left(x-2 \cos \frac{2 \pi}{5}\right)^{2}+\left(y-2 \sin \frac{2 \pi}{5}\right)^{2}+(z-0)^{2}=r_{3}^{2} \\
\left(x-2 \cos \frac{4 \pi}{5}\right)^{2}+\left(y-2 \sin \frac{4 \pi}{5}\right)^{2}+(z-0)^{2}=r_{4}^{2} \\
\left(x-2 \cos \frac{6 \pi}{5}\right)^{2}+\left(y-2 \sin \frac{6 \pi}{5}\right)^{2}+(z-0)^{2}=r_{5}^{2} \\
\left(x-2 \cos \frac{8 \pi}{5}\right)^{2}+\left(y-2 \sin \frac{8 \pi}{5}\right)^{2}+(z-0)^{2}=r_{6}^{2}
\end{array}\right.
$$

and the simplified system is

$$
\left\{\begin{array}{l}
4-0.07903587291009495 r_{4}^{2}+0.6505712626534441 r_{4} r_{5} \\
-1.3561074212644102 r_{5}^{2}-0.49301393079838474 r_{4} r_{6} \\
+2.057926246313345 r_{5} r_{6}-0.7812486085909165 r_{6}^{2}=0, \\
4 .-0.7755657991400302 r_{4}^{2}+2.0255141276190507 r_{4} r_{5} \\
-1.3257723644882415 r_{5}^{2}-0.45514735753209457 r_{4} r_{6} \\
+0.6013776619641669 r_{5} r_{6}-0.07009692145793234 r_{6}^{2}=0, \\
4 .-0.07810840035733123 r_{4}^{2}-0.23709857230188092 r_{4} r_{5} \\
-0.19856296380296357 r_{5}^{2}+0.3936762055096171 r_{4} r_{6} \\
+0.6388324706459287 r_{5} r_{6}-0.519698425132617 r_{6}^{2}=0 .
\end{array}\right.
$$

Solving (57) by the polyhedral homotopy continuation method, HOM4PS-2.0 takes 3.5 seconds and obtains a solution

$$
\begin{array}{ll}
x=1.999999999973671 & r_{1}=6.1644140029661536 \\
y=4.9999999999976644 & r_{2}=6.1024585209541776 \\
z=2.9999999999998462 & r_{3}=5.9908825202866510 \\
\dot{x}=0.9999999999990583 & r_{4}=6.1246749375685148 \\
\dot{y}=2.0000000000004783 & r_{5}=6.3136981318438636 \\
\dot{z}=3.9999999999988072 & r_{6}=6.3003888322114669,
\end{array}
$$


which has 11 correct digits.

Solving (58) by the polyhedral homotopy continuation method, HOM4PS-2.0 takes less than 0.01 seconds and obtains the solution

$r_{4}=61.246749375686868, \quad r_{5}=63.136981318440540, \quad r_{6}=63.003888322117241$,

which has 11 correct digits.

Note that the exact solutions of (57) are

$$
\begin{array}{llll}
x=20 & \dot{x}=1 & r_{1}=61.644140029689765 & r_{4}=61.24674937571382 \\
y=50 & \dot{y}=2 & r_{2}=61.02458520956943 & r_{5}=63.13698131846731 \\
z=30 & \dot{z}=4 & r_{3}=59.908825202894555 & r_{6}=63.00388832214274 .
\end{array}
$$

Table 1 lists the number of correct digits for the pentagon configuration. The first row of table is the position of the moving object. We can see that solving the original system and the simplified system by the homotopy method achieve similar accuracy. However, solving the simplified system saves a lot of cost.

TABLE 1

The number of correct digits for the pentagon configuration.

\begin{tabular}{|c|c|c|c|c|c|}
\hline & $(2,5,3)$ & $(20,50,30)$ & $(2,5,3) \times 10^{2}$ & $(2,5,3) \times 10^{3}$ & $(2,5,3) \times 10^{4}$ \\
\hline original & 13 & 11 & 9 & 8 & 5 \\
\hline simplified & 14 & 11 & 10 & 8 & 5 \\
\hline
\end{tabular}

(Two Regular Triangles): Given three stations located at $\left\{\left(2 \cos \frac{2 \pi k}{3}, 2 \sin \frac{2 \pi k}{3}\right.\right.$, $0) \mid k=0,1,2\}$ and the other three located at $\left\{\left(\cos \frac{2 \pi k}{3}, \sin \frac{2 \pi k}{3}, 0\right) \mid k=0,1,2\right\}$. We assume the object is at the position $(200,500,300)^{T}$ with the velocity $(1,2,4)^{T}$. Then the measured Doppler data should be

$$
\begin{array}{ll}
\dot{r}_{1}=3.885974155004552 & \dot{r}_{4}=3.8961310021157534 \\
\dot{r}_{2}=3.8941504270435656 & \dot{r}_{5}=3.8989284653247402 \\
\dot{r}_{3}=3.894951626856594 & \dot{r}_{6}=3.8896389634680446 .
\end{array}
$$


Hence, the original polynomial system is

$$
\left\{\begin{array}{l}
(x+2) \dot{x}+(y+2 \sqrt{3}) \dot{y}+(z-0) \dot{z}=3.885974155004552 \cdot r_{1} \\
(x-2) \dot{x}+(y-0) \dot{y}+(z-0) \dot{z}=3.8941504270435656 \cdot r_{2} \\
(x-4) \dot{x}+(y-0) \dot{y}+(z-0) \dot{z}=3.894951626856594 \cdot r_{3} \\
(x+1) \dot{x}+(y-\sqrt{3}) \dot{y}+(z-0) \dot{z}=3.8961310021157534 \cdot r_{4} \\
(x+2) \dot{x}+(y-2 \sqrt{3}) \dot{y}+(z-0) \dot{z}=3.8989284653247402 \cdot r_{5} \\
(x+1) \dot{x}+(y+\sqrt{3}) \dot{y}+(z-0) \dot{z}=3.8896389634680446 \cdot r_{6} \\
(x+2)^{2}+(y+2 \sqrt{3})^{2}+(z-0)^{2}=r_{1}^{2} \\
(x-2)^{2}+(y-0)^{2}+(z-0)^{2}=r_{2}^{2} \\
(x-4)^{2}+(y-0)^{2}+(z-0)^{2}=r_{3}^{2} \\
(x+1)^{2}+(y-\sqrt{3})^{2}+(z-0)^{2}=r_{4}^{2} \\
(x+2)^{2}+(y-2 \sqrt{3})^{2}+(z-0)^{2}=r_{5}^{2} \\
(x+1)^{2}+(y+2 \sqrt{3})^{2}+(z-0)^{2}=r_{6}^{2}
\end{array}\right.
$$

and the simplified system is

(60)

$$
\left\{\begin{array}{l}
-13.142857142857142+14.091546933694675 r_{4}^{2}-18.786077764507947 r_{4} r_{5} \\
+6.507002110304725 r_{5}^{2}-9.398837071638766 r_{4} r_{6}+5.772125889899168 r_{5} r_{6} \\
+1.8142548595411274 r_{6}^{2}=0 \\
3.1975781088237003 r_{4}^{2}-4.000351601820357 r_{4} r_{5}+1.201375786990289 r_{5}^{2} \\
-2.3958077727188263 r_{4} r_{6}+1.5984616958701363 r_{5} r_{6} \\
+0.398745390848532 r_{6}^{2}=0 \\
-16.571428571428573+20.352860593228623 r_{4}^{2}-25.09046689866869 r_{4} r_{5} \\
+7.999030346612654 r_{5}^{2}-15.622814521038018 r_{4} r_{6}+9.095792878159973 r_{5} r_{6} \\
+3.2656081881260945 r_{6}^{2}=0 .
\end{array}\right.
$$

Table 2 lists the number of correct digits for the two-triangle configuration. The first row of table is the position of the object. As in Table 2 we can see that solving the original system is slightly more accurate than solving the simplified system by the homotopy method. However, solving the simplified system is much cheaper than solving the original system.

TABLE 2

The number of correct digits for the two triangles configuration.

\begin{tabular}{|c|c|c|c|c|c|}
\hline & $(2,5,3)$ & $(20,50,30)$ & $(2,5,3) \times 10^{2}$ & $(2,5,3) \times 10^{3}$ & $(2,5,3) \times 10^{4}$ \\
\hline original & 14 & 12 & 10 & 8 & 6 \\
\hline simplified & 13 & 11 & 9 & 7 & 5 \\
\hline
\end{tabular}

(Regular Octahedron): Given six stations located at $\{( \pm 2,0,0),(0, \pm 2,0),(0$, 
$0, \pm 2)\}$. We assume the moving object is at the position $(2000,5000,3000)^{T}$ with the velocity $(1,2,4)^{T}$. Then the measured Doppler data should be

$$
\begin{array}{ll}
\dot{r}_{1}=3.8933993124376407 & \dot{r}_{4}=3.8932285531303217 \\
\dot{r}_{2}=3.893689806539583 & \dot{r}_{5}=3.8929384652559484 \\
\dot{r}_{3}=3.8926308053089875 & \dot{r}_{6}=3.8939968805122525 .
\end{array}
$$

Hence, the original polynomial system is

$$
\left\{\begin{array}{l}
(x-2) \dot{x}+(y-0) \dot{y}+(z-0) \dot{z}=3.8933993124376407 \cdot r_{1} \\
(x-0) \dot{x}+(y-2) \dot{y}+(z-0) \dot{z}=3.893689806539583 \cdot r_{2} \\
(x-0) \dot{x}+(y-0) \dot{y}+(z-2) \dot{z}=3.8926308053089875 \cdot r_{3} \\
(x+2) \dot{x}+(y-0) \dot{y}+(z-0) \dot{z}=3.8932285531303217 \cdot r_{4} \\
(x+0) \dot{x}+(y+2) \dot{y}+(z-0) \dot{z}=3.8929384652559484 \cdot r_{5} \\
(x+0) \dot{x}+(y-0) \dot{y}+(z+2) \dot{z}=3.8939968805122525 \cdot r_{6} \\
(x-2)^{2}+(y-0)^{2}+(z-0)^{2}=r_{1}^{2} \\
(x-0)^{2}+(y-2)^{2}+(z-0)^{2}=r_{2}^{2} \\
(x-0)^{2}+(y-0)^{2}+(z-2)^{2}=r_{3}^{2} \\
(x+2)^{2}+(y-0)^{2}+(z-0)^{2}=r_{4}^{2} \\
(x-0)^{2}+(y+2)^{2}+(z-0)^{2}=r_{5}^{2} \\
(x-0)^{2}+(y-0)^{2}+(z+2)^{2}=r_{6}^{2}
\end{array}\right.
$$

and the simplified system is shown as (62).

Solving (61) by the polyhedral homotopy continuation method, HOM4PS-2.0 takes 5.5 seconds, and solving (62) by the total degree homotopy continuation method, HOM4PS-2.0 takes about 0.01 seconds.

Table 3 lists the number of correct digits for the regular octahedron configuration. The first row of table is the position of the object, and " $\mathrm{x}$ " indicates the computation is out of the range that HOM4PS-2.0 can handle.

TABLE 3

The number of correct digits for the regular octahedrons configuration.

\begin{tabular}{|c|c|c|c|c|c|}
\hline & $(2,5,3)$ & $(20,50,30)$ & $(2,5,3) \times 10^{2}$ & $(2,5,3) \times 10^{3}$ & $(2,5,3) \times 10^{4}$ \\
\hline original & 13 & 11 & 10 & 8 & 5 \\
\hline simplified & 14 & 12 & 9 & $\mathrm{x}$ & $\mathrm{x}$ \\
\hline
\end{tabular}


(62)

$$
\begin{aligned}
& \left(-57253.72995800387 r_{3}+10731.495952145619 r_{3}^{3}-17887.03497970073 r_{3}^{2} r_{4}\right. \\
& +25046.15277794521 r_{3} r_{4}^{2}-7156.873445635487 r_{4}^{3}-12518.431025849644 r_{3}^{2} r_{5} \\
& -3577.243811839096 r_{3} r_{4} r_{5}+12517.694371289494 r_{3} r_{5}^{2}+3578.21707777564 r_{4} r_{5}^{2} \\
& +2.761926496344131 r_{5}^{3}-57273.82251357924 r_{6}+19677.13747668851 r_{3}^{2} r_{6} \\
& -39366.78805717656 r_{3} r_{4} r_{6}+25054.942444871875 r_{4}^{2} r_{6} \\
& -25045.648458149586 r_{3} r_{5} r_{6}-3578.4992055077487 r_{4} r_{5} r_{6} \\
& +12522.08731599397 r_{5}^{2} r_{6}+19687.182960489263 r_{3} r_{6}^{2} \\
& -17900.84788994176 r_{4} r_{6}^{2}-12527.218974045432 r_{5} r_{6}^{2} \\
& +10740.913543932473 r_{6}^{3}=0 \\
& 4096-511.8293306215535 r_{3}^{2}+47.968007553398124 r_{3}^{4}+511.876486556012 r_{3} r_{4} \\
& -95.93306310294683 r_{3}^{3} r_{4}-511.9775448544891 r_{4}^{2}+175.91172741865824 r_{3}^{2} r_{4}^{2} \\
& -95.96421325697403 r_{3} r_{4}^{3}+15.995789937200477 r_{4}^{4}+255.88098811324448 r_{3} r_{5} \\
& -47.950187618455416 r_{3}^{3} r_{5}-47.96611126900205 r_{3}^{2} r_{4} r_{5} \\
& +15.990457565858954 r_{3} r_{4}^{2} r_{5}-255.9506060265429 r_{5}^{2} \\
& +51.934831684462935 r_{3}^{2} r_{5}^{2}+47.979161478789855 r_{3} r_{4} r_{5}^{2} \\
& -15.994808112949734 r_{4}^{2} r_{5}^{2}-7.970052529113161 r_{3} r_{5}^{3}+3.998458969823517 r_{5}^{4} \\
& -767.9280624563597 r_{3} r_{6}+143.91521981580115 r_{3}^{3} r_{6}+512.0561238785305 r_{4} r_{6} \\
& -335.90545082603944 r_{3}^{2} r_{4} r_{6}+367.9504324523441 r_{3} r_{4}^{2} r_{6} \\
& -95.99789082330076 r_{4}^{3} r_{6}+255.97078668144462 r_{5} r_{6} \\
& -135.90195851620345 r_{3}^{2} r_{5} r_{6}-95.96588887754612 r_{3} r_{4} r_{5} r_{6} \\
& +15.996069237929111 r_{4}^{2} r_{5} r_{6}+95.90485185739405 r_{3} r_{5}^{2} r_{6} \\
& +47.995999228385884 r_{4} r_{5}^{2} r_{6}-7.972849529823812 r_{5}^{3} r_{6} \\
& -512.0987791064762 r_{6}^{2}+195.9589737112044 r_{3}^{2} r_{6}^{2} \\
& -336.0401770376238 r_{3} r_{4} r_{6}^{2}+176.0408328536567 r_{4}^{2} r_{6}^{2} \\
& -135.9468450894679 r_{3} r_{5} r_{6}^{2} \\
& -47.99978351595655 r_{4} r_{5} r_{6}^{2}+51.9684825149158 r_{5}^{2} r_{6}^{2}+144.0302882281241 r_{3} r_{6}^{3} \\
& -96.05094832072851 r_{4} r_{6}^{3}-47.99788039887575 r_{5} r_{6}^{3}+48.01852431706125 r_{6}^{4}=0 \\
& -0.0005438837042719413 r_{3}^{2}-1.9990702196347225 r_{3} r_{5}+1.9996141095823665 r_{5}^{2} \\
& +1.9996137284617352 r_{3} r_{6}-1.999771770948786 r_{5} r_{6} \\
& +0.00015773525706852531 r_{6}^{2}=0 \\
& -0.00035100607683435925 r_{3}^{2}+11.997105153656532 r_{3} r_{4}-11.99947370752709 r_{4}^{2} \\
& -9.995351098173611 r_{3} r_{5}+9.998070547911833 r_{5}^{2}-2.00140413612705 r_{3} r_{6} \\
& +12.001315403403057 r_{4} r_{6}-9.99885885474393 r_{5} r_{6} \\
& -0.0010532532514888615 r_{6}^{2}=0 \text {. }
\end{aligned}
$$


From Table 1, 2 and 3 we see that simplified systems for the regular pentagon and two regular triangle configurations are strongly recommended for solving the original system (1) and (2). The simplified system for the regular octahedron is recommended only when the distances between the stations and the object are not too far (less than 100 meters).

4.2. Newton's Method vs Homotopy Method. In this subsection we will compare the reliability of Newton's method and the homotopy method. Suppose six stations form a regular pentagon around a centre with radius $20 \mathrm{~m}$ :

$$
\begin{array}{ll}
\mathbf{u}_{1}=(0,0,0) & \mathbf{u}_{4}=\left(20 \cos \frac{4 \pi}{5}, 20 \sin \frac{4 \pi}{5}, 0\right) \\
\mathbf{u}_{2}=\left(20 \cos \frac{0 \pi}{5}, 20 \sin \frac{0 \pi}{5}, 0\right) & \mathbf{u}_{5}=\left(20 \cos \frac{6 \pi}{5}, 20 \sin \frac{6 \pi}{5}, 0\right) \\
\mathbf{u}_{3}=\left(20 \cos \frac{2 \pi}{5}, 20 \sin \frac{2 \pi}{5}, 0\right) & \mathbf{u}_{6}=\left(20 \cos \frac{8 \pi}{5}, 20 \sin \frac{8 \pi}{5}, 0\right) .
\end{array}
$$

Suppose the trajectories of the moving object and the associated velocity from $t=0$ to $t=100$ (seconds) are, respectively, given by

$$
\left\{\begin{array} { l } 
{ x = 1 0 0 0 0 \operatorname { s i n } ( 0 . 0 5 t - 1 ) } \\
{ y = 1 0 0 0 0 \operatorname { c o s } ( 0 . 1 t - 1 0 ) } \\
{ z = 1 0 \operatorname { e x p } ( 0 . 0 5 t + 3 ) }
\end{array} \quad \left\{\begin{array}{l}
\dot{x}=500 \cos (0.05 t-1) \\
\dot{y}=-1000 \sin (0.1 t-10) \\
\dot{z}=0.5 \exp (0.05 t+3)
\end{array}\right.\right.
$$

where the unit of the position is meter and the unit of the velocity is $\mathrm{m} / \mathrm{s}=3.6$ $\mathrm{km} / \mathrm{hr}$. The objects of the moving object of (63) are shown in Figure 1.

Denote $\mathbf{v}=(x, y, z, \dot{x}, \dot{y}, \dot{z})$. For the given data $\left\{\left(x_{j}, y_{j}, z_{j}\right)\right\}_{j=1}^{6}$ and we have, for $j=1, \ldots, 6$,

$$
\dot{r}_{j}=\frac{\left(x-x_{j}\right) \dot{x}+\left(y-y_{j}\right) \dot{y}+\left(z-z_{j}\right) \dot{z}}{\sqrt{\left(x-x_{j}\right)^{2}+\left(y-y_{j}\right)^{2}+\left(z-z_{j}\right)^{2}}}
$$

as in Section 2, thus Newton's method can be applied in solving (3).

\section{Newton's iteration:}

$$
\left\{\begin{array}{l}
\text { Given an approximate solution } \mathbf{v}^{0}, \\
\mathbf{v}^{i+1}=\mathbf{v}^{i}-D F\left(\mathbf{v}^{i}\right)^{-1} F\left(\mathbf{v}^{i}\right), \quad i=0,1,2, \cdots,
\end{array}\right.
$$

where $F=\left(F_{1}, \cdots, F_{6}\right)^{T}$ is given in (3) and $D F$ is the Jacobian of $F$.

Experiment 1: Given initial solution $\mathbf{v}(0)=(x(0), y(0), z(0), \dot{x}(0), \dot{y}(0), \dot{z}(0))^{T}$, the Doppler radar responds measured data $\left\{\dot{r}_{j}\right\}_{j=1}^{6}$ every $\Delta t$ seconds. The previous solution $\mathbf{v}\left(t_{i-1}\right)$ is taken as an initial vector of Newton's iteration for computing $\mathbf{v}\left(t_{i}\right)$. Figure 2 shows the log scale plot of the relative error for $\Delta t=0.1,0.2,0.3,0.4$, 0.5 , and 1.0. Newton's method can trace the trajectory of the moving object for $\Delta t=0.1,0.2,0.3$. For $\Delta t=0.4$ and $\Delta t=0.5, \mathbf{v}(21)$ is not in the convergent region of Newton's iteration. When $\Delta t=1.0$, Newton's iteration converges to the other solution at $t=4$.

\section{Homotopy Method:}



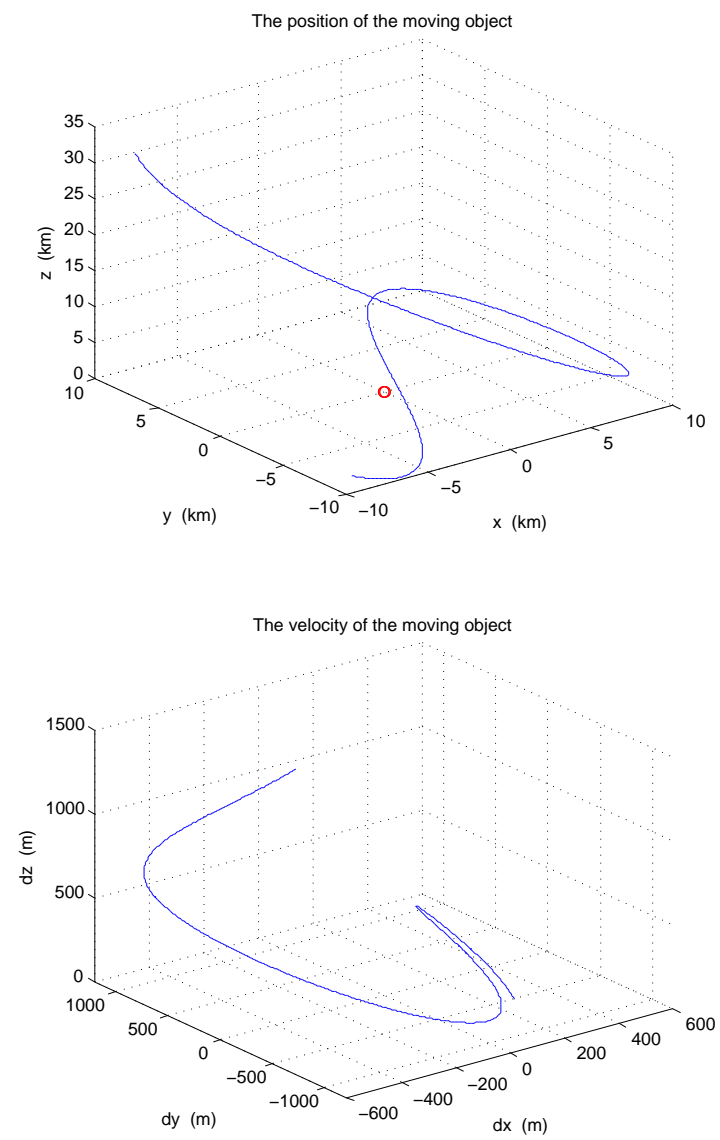

FIG. 1. the orbit of the moving object.

(i) Solving the polynomial system $\left\{q_{j}=0\right\}_{j=1}^{3}$ as in (48) by the polyhedral homotopy continuation method (Hom4ps-2.0) and get 8 solutions in variables $r_{4}, r_{5}$ and $r_{6}$.

(ii) Compute the other variables $\left\{r_{1}, r_{2}, r_{3}, x, y, z, \dot{x}, \dot{y}, \dot{z}\right\}$ by

$$
\begin{gathered}
\left\{\begin{array}{l}
r_{1}=\frac{5+\sqrt{5}}{10} \frac{\dot{r}_{4}}{\dot{r}_{1}} r_{4}-\frac{\sqrt{5}}{5} \frac{\dot{r}_{5}}{\dot{r}_{1}} r_{5}+\frac{5+\sqrt{5}}{10} \frac{\dot{r}_{6}}{\dot{r}_{1}} r_{6} \\
r_{2}=\frac{\dot{r}_{4}}{\dot{r}_{2}} r_{4}-\frac{1+\sqrt{5}}{2} \dot{\underline{r}}_{5} r_{5}+\frac{1+\sqrt{5}}{2} \frac{\dot{r}_{6}}{\dot{r}_{2}} r_{6} \\
r_{3}=\frac{1+\sqrt{5}}{2} \frac{\dot{r}_{4}}{\dot{r}_{3}} r_{4}-\frac{1+\sqrt{5}}{2} \frac{\dot{r}_{5}}{\dot{r}_{3}} r_{5}+\frac{\dot{r}_{6}}{\dot{r}_{3}} r_{6},
\end{array}\right. \\
\left\{\begin{array}{l}
\mathbf{u}=(x, y, z)^{T}=\left(V_{0}^{T} C^{T} C V_{0}\right)^{-1}\left(V_{0}^{T} C^{T} C\right) \frac{(\mathbf{n}-\mathbf{r} \circ \mathbf{r})}{2}, \\
\dot{\mathbf{u}}=(\dot{x}, \dot{y}, \dot{z})^{T}=\left(V_{0}^{T} C^{T} C V_{0}\right)^{-1}\left(V_{0}^{T} C^{T} C\right)(-\dot{R} \mathbf{r}) .
\end{array}\right.
\end{gathered}
$$

Experiment 2: The Doppler radar responds measured data $\left\{\dot{r}_{j}\right\}_{j=1}^{6}$ every $\Delta t$ seconds. We select the solution $\mathbf{v}(t)$ which is closest to $\mathbf{v}\left(t_{i-1}\right)$. Figure 3 shows the log 

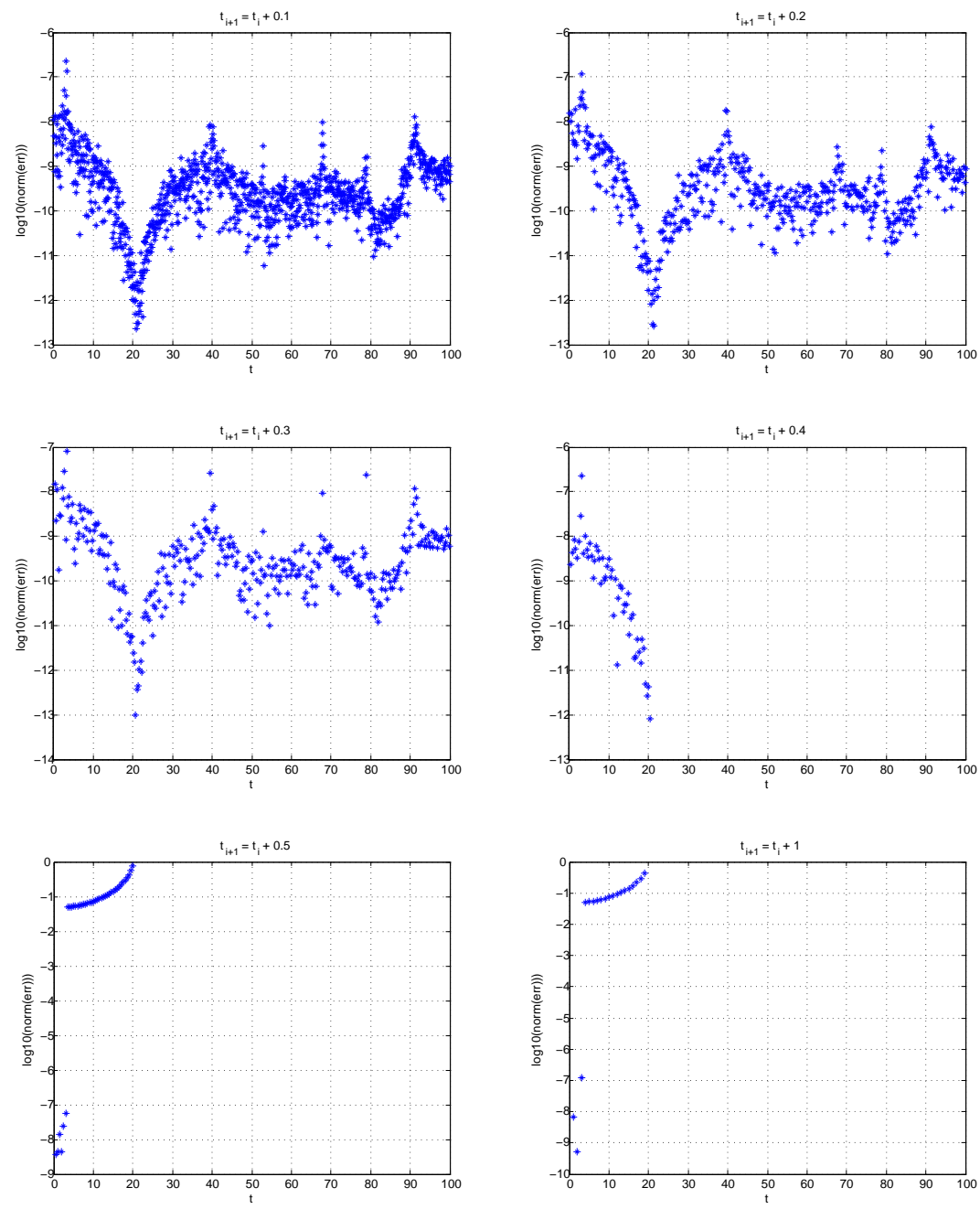

FIG. 2. The log scale plot of the relative error for Newton's method.

scale plot of the relative error for $\Delta t=0.1,0.2,0.3,0.4,0.5$, and 1.0. The polynomial homotopy method can successfully trace the trajectory of the moving object in these cases.

Remark: Newton's method can quickly compute the position of the moving object if the Doppler radar can quickly responds measured data. Otherwise, we will not have a good initial vector to start with. The polynomial homotopy method does not need any initial vectors from users. The method solves a polynomial system and outputs all solutions. Users need to choose an appropriate solution among them. If stations sit on a plane but do not form a circle, the polynomial system can be reduced to 3 independent quadratic polynomials. The reduced system is numerically stable. Although the polynomial system can be reduced to a system of 4 polynomials of de- 

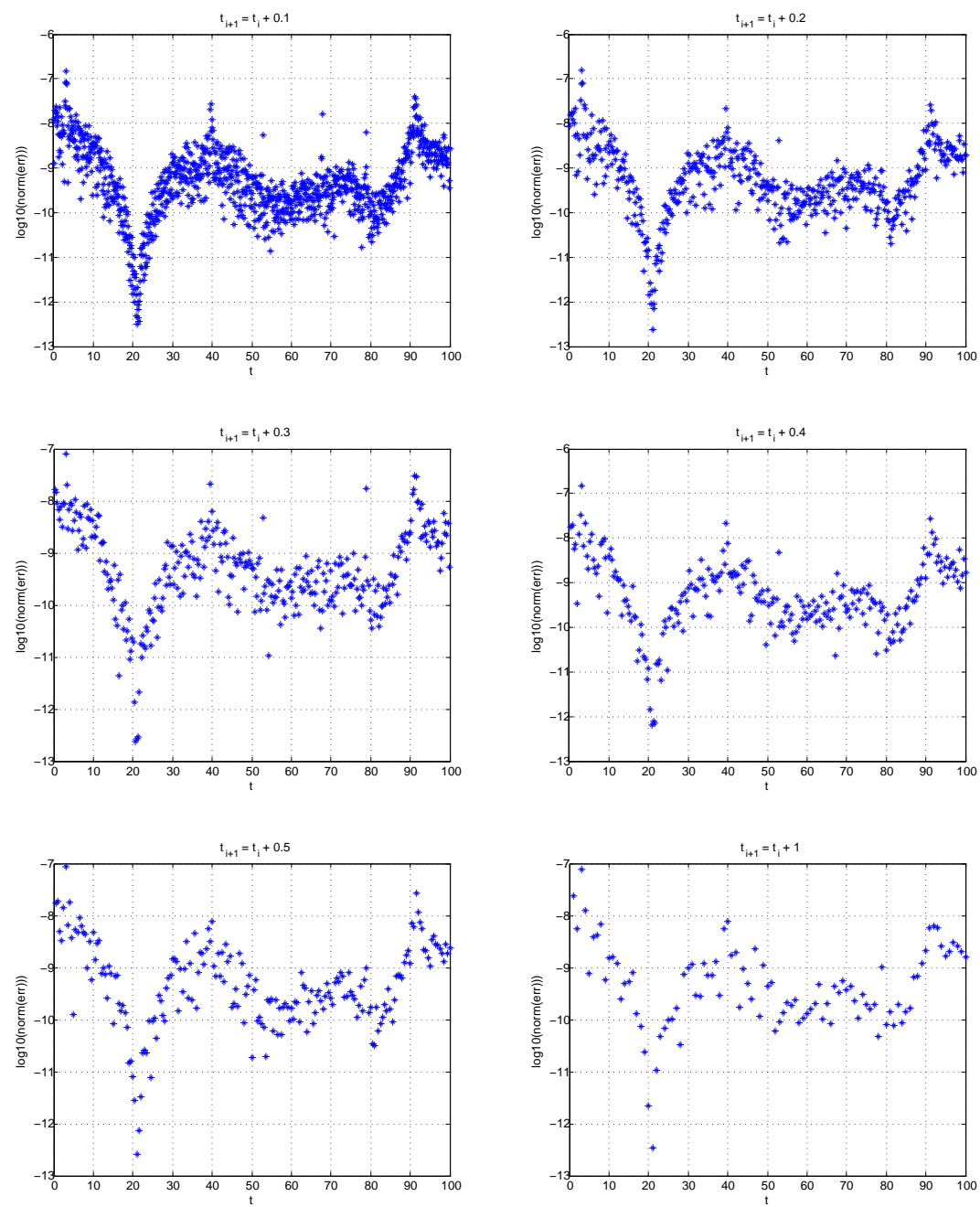

FIG. 3. The log scale plot of the relative error for the polynomial homotopy method.

grees 4, 3, 2 and 2 for the cases that stations do not sit on a plane, the reduced system may be numerical unstable. Therefore, we suggest that stations are deployed on a plane appropriately, and combine two methods into one algorithm: at the beginning, trace the position by Newton's method. Whenever the iteration fails, the polynomial homotopy method is invoked to get back solutions.

5. Conclusions. In this paper, we propose a novel simplification to reduce the original system of a moving object to a new system of only three polynomials all of degrees 2 in 3 unknowns when the 6 observation stations are located on a plane but not on a circle. Numerical results show that the homotopy method is robust and efficient for solving the simplified polynomial system when the 6 stations form a regular 
pentagon and its centre. One possible future work is to consider that whether this particular configuration of observation stations is optimal for the homotopy method.

Appendix A. For a generic $V_{0}$ we will show that $\widehat{A}$ in (16) has rank 2 . WLOG we may assume $V_{0}$ and $C V_{0}$ are of full rank. For simplicity, the spectrum of $M \in \mathbb{R}^{n \times n}$ is denoted by $\sigma(M)$. Re-write $\widehat{A}=-\widehat{C} V_{0} A+\widehat{C}=\widehat{C}\left(I-V_{0} A\right)$. Since $A V_{0}=$ $\left(V_{0}^{T} C^{T} C V_{0}\right)^{-1}\left(V_{0}^{T} C^{T} C\right) V_{0}=I_{3}, \sigma\left(A V_{0}\right)=\{1,1,1\}$. It can be shown that $\sigma\left(V_{0} A\right)=$ $\{1,1,1,0,0,0\}$ (Theorem 1.3.20 in [8]). Therefore, $\sigma\left(I-V_{0} A\right)=\{0,0,0,1,1,1\}$. It is easily seen that three columns of $V_{0}$ and the vector $\mathbf{e}=\left[\begin{array}{llllll}1 & 1 & 1 & 1 & 1 & 1\end{array}\right]^{T}$ are in the eigenspaces of $I-V_{0} A$ associated with eigenvalue 0 and 1 , respectively. Moreover, they are all in the kernel of $\widehat{A}$. Therefore, $\operatorname{rank}(\widehat{A}) \leq 6-4=2$. Now we will show that $\operatorname{rank}(\widehat{A})<2$ only when $\mathbf{u}_{2}+\mathbf{u}_{3}+\mathbf{u}_{4}+\mathbf{u}_{5}+\mathbf{u}_{6}=5 \mathbf{u}_{1}$. Let $X=\left[\begin{array}{ll}\mathbf{x}_{1} & \mathbf{x}_{2}\end{array}\right]$ whose column space $\operatorname{col}(X)$ is in the eigenspace of $I-V_{0} A$ associated with eigenvalue 1 but complemented with the space spanned by vector e. Then (i) $X \neq O$ and (ii) $\left(I-V_{0} A\right) X=X$. From (ii) we have $V_{0} A X=O$; that is, $V_{0}\left(V_{0}^{T} C^{T} C V_{0}\right)^{-1} V_{0}^{T} C^{T} C X=O$. Since $V_{0}$ and $\left(V_{0}^{T} C^{T} C V_{0}\right)^{-1}$ are of full rank, we have $V_{0}^{T} C^{T} C X=O$. Let $K=C X \in \mathbb{R}^{5 \times 2}$. Then $K \neq O_{5 \times 2}$ due to e $\notin \operatorname{col}(X)$. In addition, $C\left[\begin{array}{c}\mathbf{0}^{T} \\ K\end{array}\right]=K$ and $C\left[\begin{array}{ll}a_{1} \mathbf{e} & a_{2} \mathbf{e}\end{array}\right]=O$ for any $a_{1}, a_{2} \in \mathbb{R}$, so we can write $X=\left[\begin{array}{c}\mathbf{0}^{T} \\ K\end{array}\right]+\left[\begin{array}{ll}a_{1} \mathbf{e} & a_{2} \mathbf{e}\end{array}\right]$. Consider

$$
\widehat{A} X=\widehat{C}\left(I-V_{0} A\right) X=\widehat{C} X=\widehat{C}\left[\begin{array}{c}
\mathbf{0}^{T} \\
K
\end{array}\right]=\left[\begin{array}{ccccc}
-1 & 1 & 0 & 0 & 0 \\
-1 & 0 & 1 & 0 & 0 \\
-1 & 0 & 0 & 1 & 0 \\
-1 & 0 & 0 & 0 & 1
\end{array}\right] K
$$

From the above matrix equality, we can see that there exists a vector $\mathbf{x}_{0} \in \operatorname{col}(X)$ such that $\widehat{A} \mathbf{x}_{0}=\mathbf{0}$ (hence, $\operatorname{rank}(\widehat{A})<2$ ) if and only if $\hat{\mathbf{e}}=\left[\begin{array}{lllll}1 & 1 & 1 & 1 & 1\end{array}\right]^{T}$ is in the column space of $K$. In this case, we have $V_{0}^{T} C^{T} \hat{\mathbf{e}}=\mathbf{0}$, which is equivalent to $\mathbf{u}_{2}+\mathbf{u}_{3}+\mathbf{u}_{4}+\mathbf{u}_{5}+\mathbf{u}_{6}=5 \mathbf{u}_{1}$.

Appendix B. The system of (23) can be simplified to a system of 2 polynomials of degrees 12 and 16 with 2 unknowns. Expanding the last 2 equations in (23) yields

$$
\left\{\begin{array}{l}
a_{3} r_{3}^{2}+a_{4} r_{4}^{2}+a_{5} r_{5}^{2}+a_{6} r_{6}^{2}+a_{34} r_{3} r_{4}+a_{35} r_{3} r_{5}+\cdots+a_{56} r_{5} r_{6}+a_{0}=0 \\
b_{3} r_{3}^{2}+b_{4} r_{4}^{2}+b_{5} r_{5}^{2}+b_{6} r_{6}^{2}+b_{34} r_{3} r_{4}+b_{35} r_{3} r_{5}+\cdots+b_{56} r_{5} r_{6}+b_{0}=0
\end{array}\right.
$$


We rewrite the equations of (64) in the quadratic form: $\widetilde{\mathbf{r}}^{T} A \widetilde{\mathbf{r}}+a_{0}=0$ and $\widetilde{\mathbf{r}}^{T} B \widetilde{\mathbf{r}}+b_{0}=$ 0 , where

$$
A_{0}=\left[\begin{array}{cccc}
a_{3} & \frac{a_{34}}{2} & \frac{a_{35}}{2} & \frac{a_{36}}{2} \\
\frac{a_{34}}{2} & a_{4} & \frac{a_{45}}{2} & \frac{a_{46}}{2} \\
\frac{a_{35}}{2} & \frac{a_{45}}{2} & a_{5} & \frac{a_{56}}{2} \\
\frac{a_{36}}{2} & \frac{a_{46}}{2} & \frac{a_{56}}{2} & a_{6}
\end{array}\right], \quad B_{0}=\left[\begin{array}{cccc}
b_{3} & \frac{b_{34}}{2} & \frac{b_{35}}{2} & \frac{b_{36}}{2} \\
\frac{b_{34}}{2} & b_{4} & \frac{b_{45}}{2} & \frac{b_{46}}{2} \\
\frac{b_{35}}{2} & \frac{b_{45}}{2} & b_{5} & \frac{b_{56}}{2} \\
\frac{b_{36}}{2} & \frac{b_{46}}{2} & \frac{b_{56}}{2} & b_{6}
\end{array}\right],
$$

and $\widetilde{\mathbf{r}}=\left[\begin{array}{llll}r_{3} & r_{4} & r_{5} & r_{6}\end{array}\right]^{T}$. Since $A_{0}$ and $B_{0}$ are symmetric, we assume there is a nonsingular $V_{0}$ such that

$$
V_{0}^{T} A_{0} V_{0}=\left[\begin{array}{cccc}
\widetilde{a}_{3} & 0 & 0 & 0 \\
0 & \widetilde{a}_{4} & 0 & 0 \\
0 & 0 & \widetilde{a}_{5} & 0 \\
0 & 0 & 0 & \widetilde{a}_{6}
\end{array}\right], \quad V_{0}^{T} B_{0} V_{0}=\left[\begin{array}{cccc}
\widetilde{b}_{3} & 0 & 0 & 0 \\
0 & \widetilde{b}_{4} & 0 & 0 \\
0 & 0 & \widetilde{b}_{5} & 0 \\
0 & 0 & 0 & \widetilde{b}_{6}
\end{array}\right] .
$$

By changing variables $\left[\begin{array}{llll}r_{3} & r_{4} & r_{5} & r_{6}\end{array}\right]^{T}=V_{0}\left[\begin{array}{llll}s_{3} & s_{4} & s_{5} & s_{6}\end{array}\right]^{T}$, the system (64) becomes

$$
\left\{\begin{array}{c}
\widetilde{a}_{3} s_{3}^{2}+\widetilde{a}_{4} s_{4}^{2}+\widetilde{a}_{5} s_{5}^{2}+\widetilde{a}_{6} s_{6}^{2}+a_{0}=0 \\
\widetilde{b}_{3} s_{3}^{2}+\widetilde{b}_{4} s_{4}^{2}+\widetilde{b}_{5} s_{5}^{2}+\widetilde{b}_{6} s_{6}^{2}+b_{0}=0
\end{array}\right.
$$

Without loss of generality, we assume the matrix $\widetilde{C} \equiv\left[\begin{array}{cc}\widetilde{a}_{3} & \widetilde{a}_{4} \\ \widetilde{b}_{3} & \widetilde{b}_{4}\end{array}\right]$ is invertible. From (65) follows from

$$
\left\{\begin{array}{l}
s_{3}^{2}=c_{35} s_{5}^{2}+c_{36} s_{6}^{2}+c_{30} \\
s_{4}^{2}=c_{45} s_{5}^{2}+c_{46} s_{6}^{2}+c_{40},
\end{array}\right.
$$

where $\left[\begin{array}{ccc}c_{35} & c_{36} & c_{30} \\ c_{45} & c_{46} & c_{40}\end{array}\right]=\widetilde{C}^{-1}\left[\begin{array}{ccc}\widetilde{a}_{5} & \widetilde{a}_{6} & a_{0} \\ \widetilde{b}_{5} & \widetilde{b}_{6} & b_{0}\end{array}\right]$. Recall that the first two equations in (23) are polynomials in variables $r_{3}, r_{4}, r_{5}$ and $r_{6}$ of degree 3 and 4 , respectively. By changing variables above in the 1st equation of (23), the degrees of new equations remain the same. In fact, each of them can be written as

$$
s_{3} g_{1}+s_{4} g_{2}=s_{3} s_{4} g_{3}+g_{4},
$$

where $g_{1}, g_{2}, g_{3}$ and $g_{4}$ are polynomials in $s_{3}, s_{4}, s_{5}$ and $s_{6}$, and the exponents of $s_{3}$ and $s_{4}$ in $g_{i}$ 's are even numbers. Squaring both sides yields

$$
s_{3}^{2} g_{1}^{2}+s_{4}^{2} g_{2}^{2}+2 g_{1} g_{2} s_{3} s_{4}=s_{3}^{2} s_{4}^{2} g_{3}^{2}+2 g_{3} g_{4} s_{3} s_{4}+g_{4}^{2} .
$$

Collecting $s_{3} s_{4}$ on one side, we have $2\left(g_{1} g_{2}-g_{3} g_{4}\right) s_{3} s_{4}=s_{3}^{2} s_{4}^{2} g_{3}^{2}+g_{4}^{2}-s_{3}^{2} g_{1}^{2}-s_{4}^{2} g_{2}^{2}$. Squaring both sides of the new equation results in one equation which has variables $s_{3}, s_{4}, s_{5}$ and $s_{6}$, and the exponents of $s_{3}$ and $s_{4}$ are even numbers in all terms. By using (66), the new equation only involves $s_{5}$ and $s_{6}$. Therefore, the system (23) can be simplified to a system of two polynomials of degree 12 and 16 in two unknowns. 


\section{REFERENCES}

[1] E. L. Allgower And K. Georg, Continuation and path following, ACTA Numerica, (1993), pp. 1-64.

[2] J. W. Armstrong, Low-frequency gravitational wave searches using spacecraft Doppler tracking, Living Rev. Relativity, 9(2006).

[3] B. Bertotti, G. Comoretto, And I. Iess, Doppler tracking of spacecraft with multi-frequency links, Astron. Astrophys., 269(1993), pp. 608-616.

[4] G. H. Golub and C. F. Van Loan, Matrix Computations, 3rd ed., Johns Hopkins Univ. Press, Baltimore, MD, 1996.

[5] B. Huber And B. Sturmfels, A polyhedral method for solving sparse polynomial systems, Math. of Comp., 64(1995), pp. 1541-1555.

[6] T. Y. LI, Solving polynomial systems by the homotopy continuation method, Handbook of numerical analysis, Vol. XI, (pp. 209-304), North-Holland, Amsterdam (2003).

[7] T. L. LeE, T. Y. LI, AND C, H. TsaI, HOM4PS-2.0: A software package for solving polynomial systems by the polyhedral homotopy continuation method, Computing, 83(2008), pp. 109133.

[8] R. A. Horn and C. B. Johnson, Matrix Analysis, Cambridge University Press, 1985.

[9] S. N. Salinger and J. J. Brandstatter, Application of recursive estimation and Kalman filtering to Doppler tracking, IEEE transactions on aerospace and electronic systems,6(1970). 
TSUNG-LIN LEE ET AL. 